\title{
Robust Multichannel Blind Deconvolution via Fast Alternating Minimization
}

\author{
Filip Šroubek, Member, IEEE, and Peyman Milanfar, Fellow, IEEE
}

\begin{abstract}
Blind deconvolution, which comprises simultaneous blur and image estimation, is a strongly ill-posed problem. It is by now well-known that if multiple images of the same scene are acquired, this multichannel blind deconvolution problem is better posed and allows of blur estimation directly from the degrade images. We improve the multichannel idea by adding robustness to noise and stability in the case of large blurs or if the blur size is vastly overestimated. We formulate blind deconvolution as a $\ell_{1}$-regularized optimization problem and seek a solution by alternately optimizing with respect to the image and with respect to blurs. Each optimization step is converted to a constrained problem by variable splitting and then addressed with an augmented Lagrangian method, which permits simple and fast implementation in the Fourier domain. Rapid convergence of the proposed method is illustrated on synthetically blurred data. Applicability is also demonstrated on deconvolution of real photos taken by a digital camera.
\end{abstract}

\section{INTRODUCTION}

Image deconvolution is a classical inverse problem in image processing. Deconvolution appears in a wide range of application areas, such as photography, astronomy, medical imaging and remote sensing, just to name few. Images deteriorate during acquisition as data passes through the sensing, transmission, and recording processes. In general, the observed degradation is a result of two physical phenomena. The first is of random nature and appears in images as noise. The second is deterministic and results in blurring, which is typically modeled by convolution with some blur kernel called the point spread function (PSF). Degradation caused by convolution can thus appear in any application where image acquisition takes place. Common sources of blurring are lens imperfections, air turbulence, or camera-scene motion. Solving the deconvolution problem in a reliable way has been of prime interest in the field of image processing for several decades and has produced an enormous number of publications.

Let us first consider problems with just one degraded image, i.e. single-channel deconvolution. The simplest case is if

Copyright (C) 2010 IEEE. Personal use of this material is permitted. However, permission to use this material for any other purposes must be obtained from the IEEE by sending a request to pubs-permissions@ieee.org.

This work was supported in part by the US Air Force Grant FA955007-1-0365, the National Science Foundation Grant CCF-1016018, the Czech Ministry of Education project $1 \mathrm{M} 0572$ (Research Center DAR), and the Grant Agency of the Czech Republic project P103/11/1552. F. Śroubek accomplished this work while at the UCSC as a Fulbright Visiting Scholar.

F. Šroubek is with the Institute of Information Theory and Automation, Academy of Sciences of the Czech Republic, Pod Vodárenskou věží 4, 18208 Prague 8, Czech Republic, E-mail: sroubekf@utia.cz

P. Milanfar is with the University of California Santa Cruz, Electrical Engineering Department, Santa Cruz CA 95064 USA. E-mail: milanfar@ee.ucsc.edu the blur kernel is known (classical deconvolution problem). However even here, estimating an unknown image is illposed due to the ill-conditioned nature of the convolution operators. This inverse problem can only be solved by adopting some sort of regularization (in stochastic terms regularization corresponds to priors). Another option is to use techniques such as coded aperture [1], but this requires a modification of camera hardware, which we do not consider here. A popular recent approach is to let the unknown image be represented as a linear combination of few elements of some frame (usually an overcomplete dictionary) and force this sparse representation by using the $\ell_{p}$ norm $(0 \leq p \leq 1)$. Either we can search for the solution in the transform domain (coefficients of the frame elements), which is referred to as the synthesis approach, or regularize directly the unknown image, which is called the analysis approach. Analysis versus synthesis approach has been studied earlier [2], [3]. If the frame is an orthonormal basis, both approaches are equivalent. More interesting however is the case of redundant representation (e.g. undecimated wavelet transform), when the two approaches differ. Conclusions presented in [3] suggest that for deconvolution problems, the analysis approach is preferable, because sparsity should be enforced only on a part of the redundant representation (e.g. high-pass bands) and this can be easily implemented only in the analysis approach. Very recently it was shown that the analysis approach is solved efficiently using variable splitting and applying a Bregman iterative method [4] or augmented Lagrangian method [5] (both methods lead to the same algorithm).

If the blur kernel is unknown, we face single-channel blind deconvolution, which is clearly even more complicated than the classical deconvolution problem. This inverse problem is under-determined as we have more unknowns (image and blur) than equations. For a long time, the problem seemed too difficult to solve for general blur kernels. Past algorithms usually worked only for special cases, such as astronomical images with uniform (black) background, and their performance depended on initial estimates of PSFs. To name a few papers from this category, consider [6]-[8] and survey [9]. Probably the first attempt towards a more general blur estimation method came from Fergus et al. [10], who proposed a variational Bayesian method [11] with natural image statistics. This triggered a furious activity in the computer vision community and soon several conference papers appeared on the same topic [12]-[17]. Levin et al. [15] pointed out that the joint posterior probability of the image-blur pair favors a trivial solution of the blur being a delta function and that marginalizing the posterior (integrating out the image variable) is more 
appropriate. However, a closed form solution seldom exists and a complicated approximation of the posterior is necessary, which leads to cumbersome methods that can hardly handle large blurs. In order to avoid these drawbacks, recent methods still try to minimize directly the joint posterior probability, since it can be done in an efficient way, but perform all sorts of tricks to avoid the trivial solution. Jia [12] uses an alpha matte to extract a transparency map and estimates the blur kernel on the map. Joshi et al. [13] predicts sharp edges using edge profiles and estimates the blur kernel from the predicted edges. Cho et al. [16] applies a shock filter and gradient thresholding to restore only strong edges and estimates the blur kernel from the truncated gradient image. A similar idea further improved by a kernel refinement step, was proposed recently by $\mathrm{Xu}$ et al. [17]. In general, the single-channel blind deconvolution methods get trapped in local minima and must estimate blurs using a multiscale approach. They have many parameters that influence the result considerably and are hard to tune. The common trick for the methods to work is to have means to predict strong edges. However, if the blurry image does not have salient edges or it is corrupted by noise, all the singlechannel deconvolution methods usually fail.

The ill-posed nature of blind deconvolution can be remedied to a great extent by considering multiple images. In this case, the problem is referred to as multichannel (MC) blind deconvolution and will be the subject of our investigation. Acquired images must capture the same scene and differ only in the blur kernel. This may not seem to be easy to achieve in practice. However, the opposite is true. There are many situations where multiple images blurred in a slightly different way can be obtained. For example, if atmospheric turbulence causes blurring, we can capture several images (or video frames) in a row and due to the random nature of turbulence, each image is almost surely blurred in a different way. If camera shake causes blurring, continuous shooting (or video capture) with the camera provides several images that are blurred in a different way since our hand moves randomly. $\mathrm{MC}$ deconvolution requires that the input images are properly registered, which is one drawback compared to the singlechannel case. If the images are acquired as described above, misregistration is only minor and even simple registration methods will provide accurate and stable results; see e.g. [18] for a survey of registration methods. We will thus assume that the input images are registered up to some global translation. A simple registration method for affine transforms is used in our experiments as sketched in Sec. VI. More problematic is the occurrence of space-variant blur, which often arises in practice, such as rotating camera or profound depth of scene. We note that the method proposed here assumes a space-invariant case but by applying the method locally we can in theory deal with space-variant cases as well. We refer the interested reader to [19] and references therein for space-variant deconvolution.

One of the earliest intrinsic MC blind deconvolution methods [20] was designed particularly for images blurred by atmospheric turbulence. Harikumar et al. [21] proposed an indirect algorithm, which first estimates blur kernels and then recovers the original image by a standard nonblind method. The blur kernels are equal to the minimum eigenvector of a special matrix constructed from the blurred input images. Necessary assumptions for perfect recovery of the blurs are noise-free environment and channel coprimeness, i.e. a scalar constant is the only common factor of the blurs. Giannakis et al. [22] developed another indirect algorithm based on Bezout's identity of coprime polynomials which finds restoration filters and by convolving the filters with the input images recovers the original image. Both algorithms are vulnerable to noise and even for a moderate noise level restoration may break down. Pai et al. [23] suggested two MC restoration algorithms that, contrary to the previous two indirect algorithms, estimate directly the original image from the null space or from the range of a special matrix. Another direct method based on the greatest common divisor was proposed in [24]. In noisy cases, the direct algorithms are more stable than the indirect ones. Approaches based on the ARMA (autoregressive moving average) model are given in [25]. MC blind deconvolution using a Bussgang algorithm was proposed in [26], which performs well on spatially uncorrelated data, such as binary text images and spiky images. Sroubek et al. [27] proposed a method that reformulates Harikumar's idea in [21] as a MC regularization term and simultaneously minimizes an energy function with respect to the image and blur kernels. This allows us to handle inexact PSF sizes and compensate for small misalignments in input images, which made MC deconvolution more practical. However, small PSFs (less than $15 \times 15$ ) and images of size couple of hundreds of pixels were only considered. It is mainly because of the inefficiency of the applied numerical algorithm that the method is not converging for larger blurs and images.

Here we propose a MC blind deconvolution method that can handle very large blurs (e.g. $50 \times 50)$ and images of several Mpixels with even better accuracy and speed. The method is based on the same idea as in [27] and it is formulated as a constrained optimization problem. For image regularization, we use total variation [28] and for blur regularization we use the MC constraint proposed in [21]. We show that the original MC constraint is not robust to noise and propose a simple remedy, which requires a negligible extra computation but achieves much better stability with respect to noise. Since the optimization problem mixes the $\ell_{2}$ and $\ell_{1}$ norms, we use the state-of-the-art numerical method of augmented Lagrangian [5] to solve the blind deconvolution problem and achieve very fast convergence. As it will be clear later, positivity of blur kernels is an important constraint that must be included in the optimization problem. We show that positivity can be incorporated in augmented Lagrangian effortlessly without affecting the convergence properties.

The paper is organized as follows. Sec. II defines notation and presents the basic alternating minimization approach to blind deconvolution. Image regularization in the form of isotropic total variation is given in Sec. III. Sec. IV discusses the problem of blur estimation in the MC scenario, influence of noise and blur size, and proposes a novel blur kernel constraint with sparsity and positivity regularization. A description of the proposed algorithm is given in Sec. V together with implementation details. The experimental section VI empirically validates the proposed method and Sec. VII concludes this 
work.

\section{MC BLIND DECONVOLUTION BASICS}

We formulate the problem in the discrete domain and use frequently vector-matrix notation throughout the text. Images and PSFs are denoted by small italic letters and their corresponding vectorial representations (lexicographically ordered pixels) by small bold letters. The MC blind deconvolution problem assumes that we have $K>1$ input images $\left\{g_{1}, \ldots, g_{K}\right\}\left(g_{k}: \mathbb{N}^{2} \rightarrow \mathbb{R}\right)$ that are related to an unknown image $u: \mathbb{N}^{2} \rightarrow \mathbb{R}$ according to a model

$$
g_{k}(i)=\left(h_{k} * u\right)(i)+n_{k}(i), \quad 1 \leq k \leq K,
$$

where $h_{k}$ denotes an unknown blur (kernel or point spread function $=\mathrm{PSF}$ ) and $n_{k}$ is additive noise in the $k$-th observation. Operator $*$ stands for convolution, and $i \in \mathbb{N}^{2}$. When no ambiguity arises, we drop the multi-index $i$ from the notation. In the vector-matrix notation, (1) becomes

$$
\mathbf{g}_{k}=\mathbf{H}_{k} \mathbf{u}+\mathbf{n}_{k}=\mathbf{U h}_{k}+\mathbf{n}_{k},
$$

where matrices $\mathbf{H}_{k}$ and $\mathbf{U}$ perform convolution with $h_{k}$ and $u$, respectively. To denote the $i$-th element in the vector notation, we write $[\cdot]_{i}$, e.g., $u(i)=[\mathbf{u}]_{i}$. The size of images and blurs (matrices and vectors) will be discussed later when necessary.

In the case of multiple acquisitions, we can not expect that input images are perfectly spatially aligned. One can model such misregistation by a geometric transformation $\mathbf{W}_{k}$ that precede blurring $\mathbf{H}_{k}$, i.e., $\mathbf{H}_{k} \mathbf{W}_{k} \mathbf{u}$. If $\mathbf{W}_{k}$ is invertible, then $\mathbf{H}_{k} \mathbf{W}_{k}=\mathbf{W}_{k} \mathbf{W}_{k}^{-1} \mathbf{H}_{k} \mathbf{W}_{k}=\mathbf{W}_{k} \tilde{\mathbf{H}}_{k}$, where $\tilde{\mathbf{H}}_{k}=$ $\mathbf{W}_{k}^{-1} \mathbf{H}_{k} \mathbf{W}_{k}$. If $\mathbf{H}_{k}$ is a standard convolution with some PSF $h_{k}$ and $\mathbf{W}_{k}$ is a linear geometric transformation, then the new blurring operator $\tilde{\mathbf{H}}_{k}$ remains a standard convolution but with $h_{k}$ warped according to $\mathbf{W}_{k}$. So for linear geometric transformations (such as affine) the order of geometric transformation and blurring can be interchanged. We thus assume that input images $g_{k}$ 's can be accurately registered by linear transformations and a registration step preceding blind deconvolution removes such geometric transformations.

It is well known that the problem of estimating $u$ from $g_{k}$ 's is ill-posed, thus this inverse problem can only be solved satisfactorily by adopting some sort of regularization. Formally, this leads to the following optimization problem:

$$
\min _{u,\left\{h_{k}\right\}} F\left(u,\left\{h_{k}\right\}\right)+Q(u)+R\left(\left\{h_{k}\right\}\right),
$$

where $F$ is the data fidelity term and $Q, R$ are regularizers of the image and blurs, respectively. The formation model (1) determines the data term leading to a standard formulation $F\left(u,\left\{h_{k}\right\}\right)=\frac{\gamma}{2} \sum_{k=1}^{K}\left\|u * h_{k}-g_{k}\right\|^{2}$, where $\gamma$ is inversely proportional to the variance of noise $n_{k}$ and $\|\cdot\|$ denotes the $\ell_{2}$ norm. For simplicity, we assume the same noise variance in all frames and therefore single parameter $\gamma$ suffices. The standard approach to solve (3) is called alternating minimization and will be adopted here as well. We split the problem into two subproblems:

$$
\text { "u-step": } \min _{u} F\left(u,\left\{h_{k}\right\}\right)+Q(u)
$$

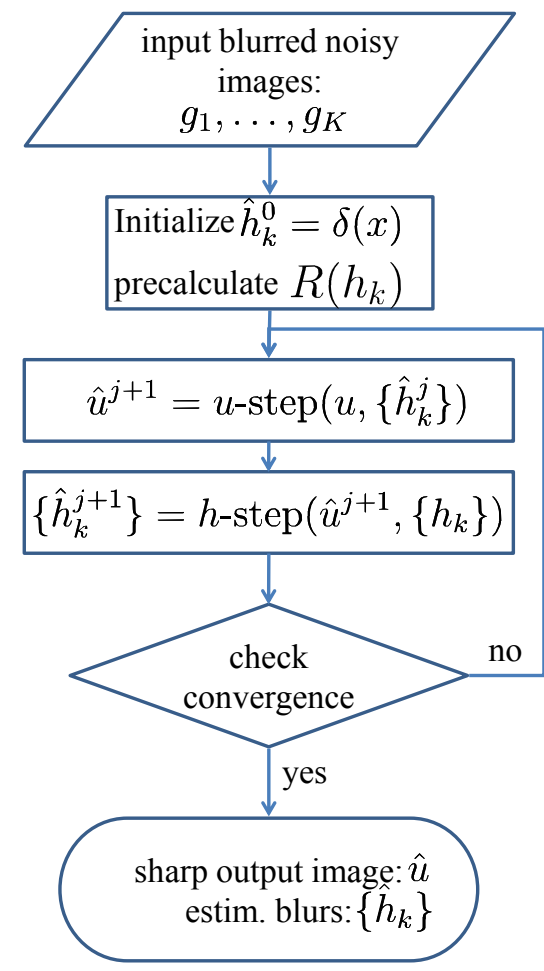

Fig. 1. Flowchart of the alternating minimization algorithm.

and

$$
\text { "h-step": } \min _{\left\{h_{k}\right\}} F\left(u,\left\{h_{k}\right\}\right)+R\left(\left\{h_{k}\right\}\right),
$$

and alternate between them; see an algorithm flowchart in Fig. 1. Convergence to the global minimum is theoretically not guaranteed since the unknown variables are coupled in the data term $F$. However, we show that each subproblem separately converges to its global minimum and that it can be solved efficiently by the augmented Lagrangian method (ALM). This implies that in general the global minimum of (3) is attainable after few alternations between the subproblems. The next two sections describe in detail the image $Q$ and blur $R$ regularization terms.

\section{IMAGE REGULARIZATION}

A popular recent approach to image regularization is to assume that the unknown image $u$ is represented as a linear combination of few elements of some frame (usually an overcomplete dictionary) and force this sparse representation by using the $\ell_{1}$ norm (or $\ell_{0}$ ). Arguably, the best known and most commonly used image regularizer, which belongs to the category of sparse priors, is the total variation (TV) norm [28].

The isotropic TV model is the $\ell_{1}$ norm of image gradient magnitudes and takes the form

$$
Q(u)=\sum_{i} \phi(\nabla u(i))=\sum_{i} \sqrt{\left(\nabla_{x} u(i)\right)^{2}+\left(\nabla_{y} u(i)\right)^{2}},
$$

where $\phi(x)=\|x\|$. The TV regularizer thus forces the solution to have sparse image gradient. Depending on the type of data, one can have sparsity in different domains. This modification is however easy to achieve. All we have to do is to replace 
derivatives with a transformation (e.g. wavelet-like multi-scale transform), which gives sparse representation of our data.

Using the vector matrix notation, the isotropic TV (6) can be written as

$$
Q(\mathbf{u})=\Phi\left(\mathbf{D}_{x} \mathbf{u}, \mathbf{D}_{y} \mathbf{u}\right)=\sum_{i} \sqrt{\left[\mathbf{D}_{x} \mathbf{u}\right]_{i}^{2}+\left[\mathbf{D}_{y} \mathbf{u}\right]_{i}^{2}}
$$

where $\mathbf{D}_{x}$ and $\mathbf{D}_{y}$ are matrices performing derivatives with respect to $x$ and $y$, respectively.

\section{BLUR ESTIMATION AND REGULARIZATION}

We first review a multichannel (MC) PSF estimation method proposed in [21], [22], which was later used in MC blind deconvolution as PSF regularizer [27]. We demonstrate that the method is not robust to noise and show a novel improvement in this aspect. To keep the notation simple, let us assume 1D data and a two-channel convolution model $(1)(K=2)$. The following discussion can be easily extended to $2 \mathrm{D}$ data and any $K>2$. The size of $1 \mathrm{D}$ data $g_{k}, u$ and $h_{k}$ is $M, N$ and $L$, respectively, with $N \gg L$. Noise $n_{k}$ is of the same size as $g_{k}$. Kernels $h_{k}$ 's can be of different size but we can always pad the smaller ones with zeros to have the size of the largest one and therefore $L$ refers to the size of the largest PSF. To deal correctly with convolution at image boundaries, we work with convolution that returns a "valid" part of the support and thus $M=N-L+1$. The matrices $\mathbf{H}_{k}$ and $\mathbf{U}$ in the vector-matrix formation model (2) are thus of size $M \times N$ and $M \times L$, respectively.

Let $\hat{h}_{k}$ be an estimate of $h_{k}$. In general, the original PSF size $L$ is not known and therefore $\hat{h}_{k}$ can be of different size denoted here as $\hat{L}$. Let us study 3 cases that will be used in the following discussion: (a1) noiseless case $\left(n_{k}=0\right),(\mathrm{a} 2)$ PSF size is exactly known $(\hat{L}=L)$, and (a3) original PSFs are weakly coprime and images $g_{k}$ 's are persistently exciting for size $L$. A set of kernels $\left\{h_{k}\right\}$ is called weakly coprime [22], if there exists a kernel $s$ and a set $\left\{\tilde{h}_{k}\right\}$ so that $\forall k$, $h_{k}=s * \tilde{h}_{k}$, then $s$ is a scalar. In other words, if the kernels are decomposable they must not have a common kernel. An image $z$ of size $M$ is called persistently exciting [21] for size $L$, if its "valid" convolution matrix $\mathbf{Z}$ of size $(M-L+1) \times L$ has full column rank. Note that such an image will be also persistently exciting for any size smaller than $L$.

\section{A. The noiseless case}

We first consider a situation, when all three assumptions (a1), a(2), and (a3) hold. If $\hat{h}_{k}=h_{k}$ then

$$
g_{2} * \hat{h}_{1}-g_{1} * \hat{h}_{2}=h_{2} * u * \hat{h}_{1}-h_{1} * u * \hat{h}_{2}=0,
$$

where we used the commutative property of convolution. Rewriting the above relation in the vector-matrix notation, we get

$$
\left[\mathbf{G}_{2},-\mathbf{G}_{1}\right] \mathbf{h}=\mathbf{0}
$$

where $\mathbf{h}=\left[\mathbf{h}_{1}^{T}, \mathbf{h}_{2}^{T}\right]^{T}$. Matrices $\mathbf{G}_{1}$ and $\mathbf{G}_{2}$ denote "valid" convolution with $g_{1}$ and $g_{2}$, respectively, and they are of size $(M-L+1) \times L$. Note that in the case of $K>2$, it is sufficient to consider all unordered pairs of images, which is equal to the combinatorial number $\left(\begin{array}{c}K \\ 2\end{array}\right)$. Thus, for example for $K=3$ the number of image pairs is $\left(\begin{array}{l}3 \\ 2\end{array}\right)=3$, (9) becomes

$$
\left[\begin{array}{ccc}
\mathbf{G}_{2} & -\mathbf{G}_{1} & \mathbf{0} \\
\mathbf{G}_{3} & \mathbf{0} & -\mathbf{G}_{1} \\
\mathbf{0} & \mathbf{G}_{3} & -\mathbf{G}_{2}
\end{array}\right] \mathbf{h}=\mathbf{0} .
$$

Let us continue with $K=2$ and define a symmetric, positive semidefinite, $2 L \times 2 L$ matrix

$$
\mathbf{R}=\left[\mathbf{G}_{2},-\mathbf{G}_{1}\right]^{T}\left[\mathbf{G}_{2},-\mathbf{G}_{1}\right] .
$$

The computational complexity of constructing this matrix is discussed in Sec.V-C. It follows from (9) that the correct estimates of $h_{k}$ lie in the null space of $\mathbf{R}$. We refer to eigenvalues of $\mathbf{R}$ as $\lambda_{i}$ 's $\left(\lambda_{1}<\lambda_{2}<\ldots<\lambda_{2 L}\right)$ and the corresponding eigenvectors as $\mathbf{v}_{i}$ 's. Since (a2) and (a3) hold, $\mathbf{R}$ has exactly one zero eigenvalue $\lambda_{1}$ and the eigenvector $\mathbf{v}_{1}$ is equal to the correct PSFs $h_{k}$ stacked in one vector multiplied by a scalar. Note that $\mathbf{R}$ is constructed solely from the input images $\mathbf{g}_{k}$ 's and it can be thus used for PSF estimation. An example of $\mathbf{R}$ spectrum (plot of $\lambda_{i}$ 's) is in Fig. 2(a) (solid line). The matrix $\mathbf{R}$ was constructed from images blurred by two $5 \times 5$ PSFs in Fig. 3(a). Notice the prominent kink at the first eigenvalue $\lambda_{1}$. The corresponding eigenvector $\mathbf{v}_{1}$ represents exactly the original PSFs. This fact is also illustrated in Fig. 4(a), which plots the representation $\left\{\alpha_{i}\right\}$ of $\mathbf{h}$ in the basis $\left\{\mathbf{v}_{i}\right\}$, i.e.h $=\sum_{i=1}^{2 L} \alpha_{i} \mathbf{v}_{i}$. One can use $\mathbf{R}$ to build a quadratic form

$$
R(\mathbf{h})=\mathbf{h}^{T} \mathbf{R h}
$$

and rewrite the $\mathbf{v}_{1}$ eigenvector estimation as a constrained optimization problem

$$
\min _{\mathbf{h}} R(\mathbf{h}) \quad \text { s.t. } \quad \forall k \sum_{i} h_{k}(i)=1 .
$$

As proposed in [27], it is better to use the quadratic term $R$ as a PSF regularization term in the blind MC deconvolution problem (3). Because of the favorable spectrum of $\mathbf{R}$, convergence of such algorithms is very fast.

\section{B. The noisy case}

Let us see what happens if we remove (a1) and allow noise to enter the formation model (1). We assume uncorrelated normally distributed noise, $n_{k} \sim N\left(0, \sigma^{2}\right)$. It follows from (2) that the convolution matrices $\mathbf{G}_{k}$ 's in (9) take the form

$$
\mathbf{G}_{k}=\mathbf{H}_{k} \mathbf{U}+\mathbf{N}_{k},
$$

where this time $\mathbf{H}_{k}$ is of size $(M-L) \times M$ and $\mathbf{N}_{k}$ is a noise convolution matrix constructed in the same way as $\mathbf{G}_{k}$ but using elements of $n_{k}$ instead of $g_{k}$. Substituting for $\mathbf{G}_{k}$ in (9), we get

$$
\left[\mathbf{G}_{2},-\mathbf{G}_{1}\right] \mathbf{h} \sim N(\mathbf{0}, \Sigma),
$$

where $\Sigma=\operatorname{cov}\left(\mathbf{H}_{2} \mathbf{U h}_{1}+\mathbf{N}_{2} \mathbf{h}_{1}-\mathbf{H}_{1} \mathbf{U h}_{2}-\mathbf{N}_{1} \mathbf{h}_{2}\right)=$ $\operatorname{cov}\left(\mathbf{N}_{2} \mathbf{h}_{1}-\mathbf{N}_{1} \mathbf{h}_{2}\right)=\operatorname{cov}\left(\mathbf{H}_{1} \mathbf{n}_{2}-\mathbf{H}_{2} \mathbf{n}_{1}\right)=\mathbf{H}_{1} \operatorname{cov}\left(\mathbf{n}_{2}\right) \mathbf{H}_{1}^{T}+$ $\mathbf{H}_{2} \operatorname{cov}\left(\mathbf{n}_{1}\right) \mathbf{H}_{2}^{T}=\sigma^{2}\left(\mathbf{H}_{1} \mathbf{H}_{1}^{T}+\mathbf{H}_{2} \mathbf{H}_{2}^{T}\right)$, since $h_{2} * u * h_{1}=$ $h_{1} * u * h_{2} \Longleftrightarrow \mathbf{H}_{2} \mathbf{U h}_{1}=\mathbf{H}_{1} \mathbf{U h}_{2}$, which follows from (8). Because of noise, we cannot expect that the smallest eigenvalue of $\mathbf{R}$ will be zero anymore. Indeed, the kink 
visible in the noiseless case is completely leveled out in the noisy case. Fig. 2(a) (dotted line) shows the spectrum of $\mathbf{R}$ for the input data used before but corrupted by noise with $\mathrm{SNR}=40 \mathrm{~dB}$, which is a relatively small level of noise hardly detectable by human eyes. Eigenvector $\mathbf{v}_{1}$ is not informative any more and represents an erroneous solution as shown in Fig. 3(b). The correct solution is a linear combination of all eigenvectors with the weights almost randomly distributed as seen in Fig. 4(b).

The maximum-likelihood estimation of kernels must include the covariance matrix $\Sigma$ in $\mathbf{R}$, i.e.,

$$
\mathbf{R}_{\Sigma}=\left[\mathbf{G}_{2},-\mathbf{G}_{1}\right]^{T} \Sigma^{-1}\left[\mathbf{G}_{2},-\mathbf{G}_{1}\right] .
$$

The spectrum of $\mathbf{R}_{\Sigma}$ retains the kink at the first smallest eigenvalue $\lambda_{1}$ as Fig. 2(b) (solid line) shows. For comparison, we show the original spectrum of $\mathbf{R}$ in (10) as a dotted line (also in Fig. 2(a)). Eigenvector $\mathbf{v}_{1}$ of $\mathbf{R}_{\Sigma}$ captures the original PSFs as shown in Fig. 3(c). Encoding of the true kernels $\mathbf{h}$ in the basis $\left\{\mathbf{v}_{i}\right\}$ is relatively sparse and cluster around the smallest eigenvalues; see Fig. 4(c). The same behavior persists even for much higher noise levels (around 10dB). The construction of $\mathbf{R}_{\Sigma}$ has one severe drawback: we must know the correct kernels $h_{k}$ 's a priori in order to build $\Sigma$. Since our aim is to estimate PSFs this seem to be contradictory. One can apply an iterative procedure and update $\Sigma$ with every new estimate of $h_{k}$ 's as proposed in [21]. Unfortunately, this framework is not guaranteed to converge. In addition, inversion of $\Sigma$ can be very costly, which makes the whole calculation of $\mathbf{R}_{\Sigma}$ for large kernels (large $L$ ) impossible.

We propose to filter the blurred input images $g_{k}$ 's in such a way, so that $\mathbf{R}$ without $\Sigma$ in (10) will be close to $\mathbf{R}_{\Sigma}$ in (15). If we filter the input images with some kernel $p$, then

$$
\left[\mathbf{P G}_{2},-\mathbf{P G}_{1}\right] \mathbf{h} \sim N\left(\mathbf{0}, \Sigma_{\mathbf{P}}\right),
$$

where $\mathbf{P}$ performs convolution with $p$ and the covariance matrix is $\Sigma_{\mathbf{P}}=\sigma^{2}\left(\mathbf{H}_{1} \mathbf{P P}^{T} \mathbf{H}_{1}^{T}+\mathbf{H}_{2} \mathbf{P} \mathbf{P}^{T} \mathbf{H}_{2}^{T}\right)=\sigma^{2} \mathbf{P}\left(\mathbf{H}_{1} \mathbf{H}_{1}^{T}+\right.$ $\left.\mathbf{H}_{2} \mathbf{H}_{2}^{T}\right) \mathbf{P}^{T}$. The best choice of the filter $p$ is such that $\Sigma_{\mathbf{P}}=\sigma^{2} \mathbf{I}$, since then the covariance matrix can be neglected. However, this would again require a priori knowledge of unknown kernels $h_{k}$ 's, since $p$ depends on $h_{k}$ 's. Achieving a diagonal correlation matrix means that we want to spatially decorrelate the blur kernels. In the absence of any prior knowledge of the blurs, we wish to employ a decorrelation method that is sufficiently general. As such, given the wellaccepted assumption of sparsity on high frequency spatial structures, the natural choice is to apply a Laplacian operator. The justification is therefore empirical, but quite reasonable we believe. In Fig. 5(a) we show a small part of the covariance matrix $\Sigma$ for our example with two blurs and in Fig. 5(b) the covariance matrix $\Sigma_{\mathbf{P}}$ with $\mathbf{P}$ being the Laplacian. The covariance matrix of the filtered images is not diagonal but close to diagonal. The Laplacian produces images, which are relatively sparse and therefore spatially uncorrelated to a great extent. The same holds for PSFs that blur the images, which accounts for the close-to-diagonal covariance matrix.

Let $\Delta$ denote a matrix that performs convolution with a discrete Laplacian kernel $l$ (in 1D $l=[1,-2,1]$ ). The

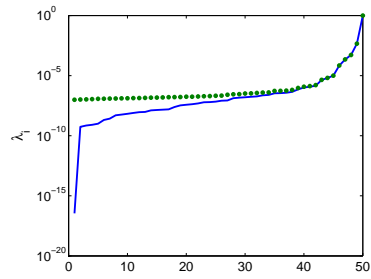

(a)

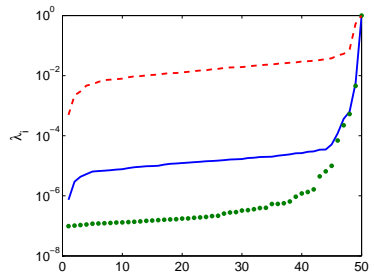

(b)
Fig. 2. Spectra of kernel regularization matrices $\mathbf{R}$ in (10), $\mathbf{R}_{\Sigma}$ in (15), and $\mathbf{R}_{\Delta}$ in (17): (a) $\mathbf{R}$ in the noiseless (solid line) and noisy case (dotted line); (b) $\mathbf{R}_{\Sigma}$ (solid line) and $\mathbf{R}_{\Delta}$ (dashed line) in the noisy case.

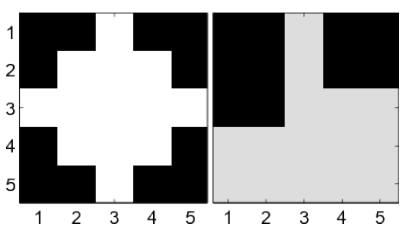

(a)

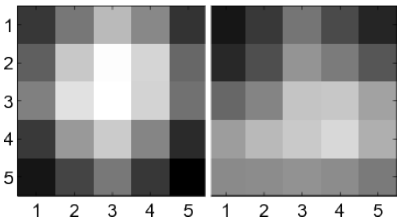

(c)

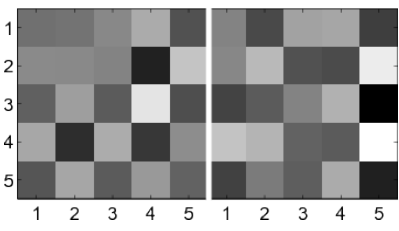

(b)

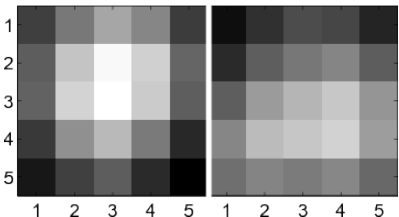

(d)
Fig. 3. Point Spread Functions and their estimates (first eigenvectors) in the noisy case: (a) two original PSFs of size $5 \times 5$, (b) estimation using $\mathbf{R}$, (c) estimation using $\mathbf{R}_{\Sigma}$, (d) estimation using $\mathbf{R}_{\Delta}$.

proposed modification of the matrix $\mathbf{R}$ is

$$
\mathbf{R}_{\Delta}=\left[\Delta \mathbf{G}_{2},-\Delta \mathbf{G}_{1}\right]^{T}\left[\Delta \mathbf{G}_{2},-\Delta \mathbf{G}_{1}\right] .
$$

The matrix $\mathbf{R}_{\Delta}$ depends only on the input images $g_{k}$ 's and the construction is trivial. The spectrum of this matrix retains the kink (Fig. 2(b), dashed line) and relatively sparse representation of $\mathbf{h}$ as shown in Fig. 4(d). The eigenvector $\mathbf{v}_{1}$ estimates $h_{k}$ 's in a similar way as the ideal $\mathbf{R}_{\Sigma}$, see Fig. 3(d).

\section{Overestimated kernel size}

It is unrealistic to assume that the kernel size $L$ is exactly known in practice. Let us thus consider the case when both (a1) and (a3) hold but (a2) is violated with the kernel size being overestimated, i.e., $\hat{L}>L$. We can readily see that if $\hat{h}_{k}=s * h_{k}$, where $s$ is an arbitrary spurious kernel of size $S=\hat{L}-L+1$, the multichannel constraint (8) still holds

$$
g_{2} * \hat{h}_{1}-g_{1} * \hat{h}_{2}=h_{2} * u * s * h_{1}-h_{1} * u * s * h_{2}=0 .
$$

In the language of matrix eigenvalues and eigenvectors this fact translates as follows. The matrix $\mathbf{R}$ defined in (10) is of size $\hat{L} \times \hat{L}$. The correct kernels lie again in the null space of $\mathbf{R}$, but this time the matrix nullity is of the size of the spurious kernel, i.e., nullity $(\mathbf{R})=S$. The regularization term (11) built from $\mathbf{R}$ becomes less restrictive (more "flat") because of the increased nullity. Therefore, convergence of any minimization algorithm, which estimates PSFs using the proposed regularizer $R$, is seriously hindered in the case of overestimated kernel size. Note that if the kernel size 


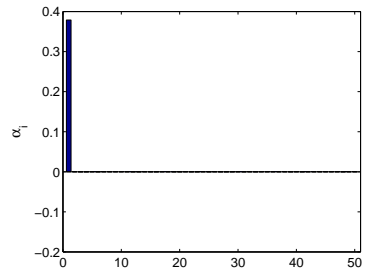

(a)

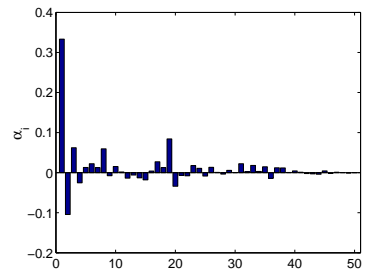

(c)

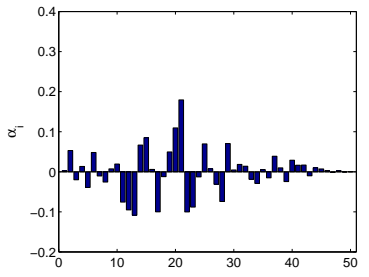

(b)

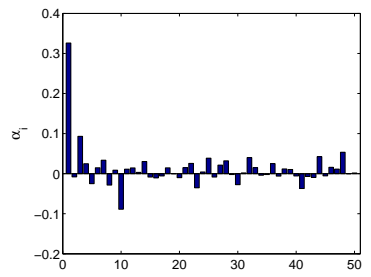

(d)
Fig. 4. Representation of PSFs in the eigenvector basis of regularization matrices: (a) $\mathbf{R}$ in the noiseless case, (b) $\mathbf{R}$ in the noisy case, (c) $\mathbf{R}_{\Sigma}$ in the noisy case, (d) $\mathbf{R}_{\Delta}$ in the noisy case.

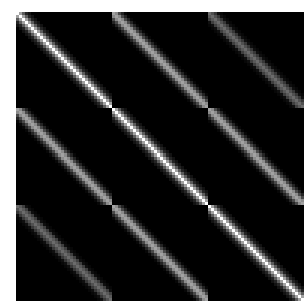

(a)

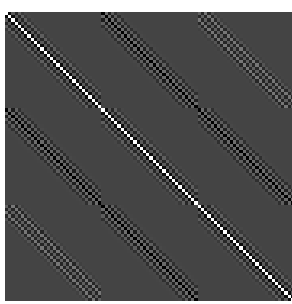

(b)
Fig. 5. Covariance matrices: (a) calculated from the original PSFs, (b) calculated from the Laplacian of PSFs.

$\hat{L}$ is underestimated, (18) does not hold anymore and we cannot estimated the kernels at all. We will not consider the underestimated case and instead focus on improving the stability of the overestimated case.

One can be tempted to assume that the unconstrained optimization problem as defined in (5) would eliminate the ambiguity inherent in $R(\mathbf{h})$. Using the vector-matrix notation, this problem rewrites as

$$
\min _{\mathbf{h}} \frac{\gamma}{2} \sum_{k=1}^{2}\left\|\hat{\mathbf{U}} \mathbf{h}_{k}-\mathbf{g}_{k}\right\|^{2}+R(\mathbf{h})
$$

where $\hat{\mathbf{U}}$ is $M \times \hat{L}$ convolution matrix with the estimate $\hat{u}$ of the original image $u$. If the estimate $\hat{u}=u$, the above optimization problem is well-posed and in fact we do not need the regularizer $R$ at all. However, this scenario is unrealistic, since we do not know the original image. Alternating minimization often starts with $\hat{u}$ equal to a so-called average image, i.e., $\hat{u}=\frac{1}{K} \sum_{k} g_{k}$. To illustrate the behavior of the data term $F\left(\hat{u},\left\{\hat{h}_{k}\right\}\right)$ with respect to the spurious kernel $s$, we conducted the following experiment. We generated two blurry signals $g_{1}$ and $g_{2}$ using some random positive PSFs $h_{1}$ and $h_{2}$ of size $L$. We set $\hat{L}=L+1$ so the spurious kernel $s$ is of size $2, s=\left[s_{1}, s_{2}\right]$. Let us consider kernels of the form $\hat{h}_{k}=s * h_{k}$ that preserve energy $\sum_{i} \hat{h}_{k}(i)=1$, then $R(\hat{\mathbf{h}})=0$ for any $s$ and $s_{2}=1-s_{1}$. The data term
$F\left(\hat{u}, \hat{h}_{k}\right)$ with $\hat{u}$ being the average image is a function of $s_{1}$ and we plot its values for different $s_{1}$ in Fig. 6. The minimum is reached for a negative value of $s_{1}$ and the same behavior was observed for any pair of blurs $h_{1}, h_{2}$. The data term is thus biased towards kernels with small negative values and the unconstrained optimization problem (19) is inappropriate if the kernel size is overestimated. An intuitive explanation is the following. Since we use the average image, the value of $F$ would reach its minimum for some $\hat{h}_{k}$ 's close to delta functions. Such a solution is however heavily penalized by $R$, which allows only PSFs of the form $s * h_{k}$. In order to get closer to the delta-function solution, $s$ must act as an inverse filter to all positive $h_{k}$ and this means that it must perform differentiation and hence negative values in $s$ are inevitable.

Forcing positivity on kernels is the remedy to the above problem. Clearly this approach is possible only for positive kernels. We encounter positive-only kernels in many deconvolution problems and making this assumption is thus not very restrictive. With the positivity constraint the above problem can be solved by means of quadratic programming. Here we show a different approach, which will allow us an elegant integration in the ALM and much faster implementation than quadratic programming. We have empirically observed that forcing sparsity on $h_{k}$ further boosts convergence. In order to guarantee both positivity and sparsity, we propose to use a new kernel regularizer

$$
R(\mathbf{h})=\frac{\delta}{2} \mathbf{h}^{T} \mathbf{R}_{\Delta} \mathbf{h}+\Psi(\mathbf{h}),
$$

where

$$
\Psi(\mathbf{h})=\sum_{k=1}^{K} \sum_{i=1}^{\hat{L}} \psi\left(h_{k}(i)\right), \quad \psi(t)= \begin{cases}t & \text { if } t \geq 0 \\ +\infty & \text { otherwise }\end{cases}
$$

and $\delta$ is the weight that controls the influence of the MC constraint $\mathbf{R}_{\Delta}$. The definition of $\psi$ ensures positivity by absolutely penalizing negative values and forces sparsity by calculating the $\ell_{1}$ norm of positive kernels.

Note that it is not necessary to explicitly include the constraint $\forall k \sum_{i} h_{k}(i)=1$ as in (12), which preserves the average gray value in images. This constraint is automatically enforced by the fidelity term $\sum_{k=1}^{2}\left\|u * h_{k}-g_{k}\right\|$ in (19). If the mean value of the estimated image $u$ is equal to the mean value of $g_{k}$, then by solving (19) ( $h$-step) we always preserve $\sum_{i} h_{k}(i)=1$. The $u$-step in (4) does not change the mean value of $u$ either because the fidelity term is present there as well. Therefore the condition is not modified in alternating minimization and we only have to guarantee that initial PSFs follow the constraint.

\section{Kernel coprimeness}

Let us consider the assumption (a3) of persistently exciting images and weakly coprime kernels. The condition of persistently exciting image is a very mild one. Usually $M \gg L$, convolution matrices $\mathbf{G}_{k}$ 's have many more rows than columns and the probability that the matrices will not have full column rank is thus very small. We do not consider here degenerate 


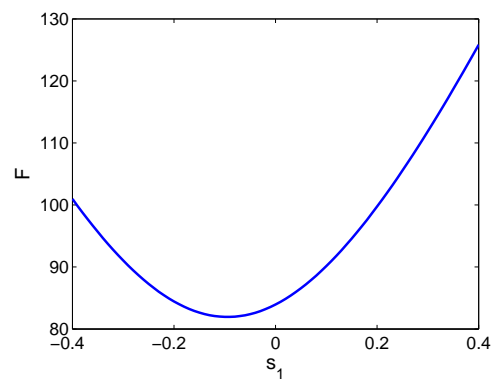

Fig. 6. The data term $F\left(\hat{u},\left\{s * h_{k}\right\}\right)$ as a function of the first elements $s_{1}$ of the $2 \times 1$ spurious vector $s=\left[s_{1}, s_{2}\right]$, where $s_{2}=1-s_{1}$. The minimum is not reached for $s=[0,1]$ (delta function) but for $s$ with a small negative value.

cases, such as perfectly uniform or periodic images, that may not be persistently exciting.

The condition of weakly coprime kernels may seem to be more problematic. In the $1 \mathrm{D}$ case (signals), any kernel of length $L$ can be decomposed (factorized) into $L-1$ kernels (root factors) of size 2, which is the direct consequence of the Fundamental Theorem of Algebra ${ }^{1}$; see for example [29]. It is therefore likely that there might exist a factor common to all kernels $h_{k}$ 's. In the 2D case (images), no such factorization in general exists and as also discussed in [21], coprimeness holds deterministically for most of the $2 \mathrm{D}$ cases of practical interest.

If the common factor exists despite its low probability, kernel estimation still partially works. We are able to recover kernels without their common factor and the common factor remains as a blur in the estimated image.

\section{OPTIMIZATION ALGORITHM}

Alternating minimization, which solves the MC blind deconvolution problem (3), consists of two subproblems: minimization with respect to the image ( $u$-step) and the minimization with respect to the blurs ( $h$-step). Both subproblems share some similarities, because both the image (7) and blur regularizer (20) are not smooth and introduce nonlinearity in the problem. Direct minimization in each step would be thus a slow process. A simple procedure that solves such problems is called variable splitting, which decouples the $\ell_{2}$ and $\ell_{1}$ portions of the problem (3) by introducing auxiliary variable and converting each subproblem to two simpler minimization steps. We then apply the ALM, which is equivalent to the split Bregman iterative method [4], to solve the subproblems. Our derivation follows the work presented in [5] and partially in [4]. Unique aspects of our algorithm will be emphasized. From now on, we will exclusively use the vector-matrix notation and stack all observations into one system by using compact notation $\mathbf{g}=\left[\mathbf{g}_{1}^{T}, \ldots, \mathbf{g}_{K}^{T}\right]^{T}, \mathbf{h}=\left[\mathbf{h}_{1}^{T}, \ldots, \mathbf{h}_{K}^{T}\right]^{T}$, $\mathbf{H}=\left[\mathbf{H}_{1}^{T}, \ldots, \mathbf{H}_{K}^{T}\right]^{T}$, and the convolution matrix $\mathbf{U}$ will now denote a block diagonal matrix with $K$ blocks, where each block is the original $\mathbf{U}$ from (2).

\footnotetext{
${ }^{1}$ However, some of the factors may contain complex values.
}

\section{A. U-step}

Using the TV regularizer (7), minimization with respect to the image (4) writes as

$$
\min _{\mathbf{u}} \frac{\gamma}{2}\|\mathbf{H u}-\mathbf{g}\|^{2}+\Phi\left(\mathbf{D}_{x} \mathbf{u}, \mathbf{D}_{y} \mathbf{u}\right)
$$

Applying variable splitting, we replace $\mathbf{D}_{x} \mathbf{u}$ by $\mathbf{v}_{x}$ and $\mathbf{D}_{y} \mathbf{u}$ by $\mathbf{v}_{y}$. This yields a constrained problem

$\min _{\mathbf{u}, \mathbf{v}_{x}, \mathbf{v}_{y}} \frac{\gamma}{2}\|\mathbf{H u}-\mathbf{g}\|^{2}+\Phi\left(\mathbf{v}_{x}, \mathbf{v}_{y}\right) \quad$ s.t. $\quad \mathbf{v}_{x}=\mathbf{D}_{x} \mathbf{u}, \mathbf{v}_{y}=\mathbf{D}_{y} \mathbf{u}$,

which is equivalent to (22). The ALM (or split-Bregman iteration) tackles the constrained problem (23) by considering a functional

$$
\begin{aligned}
& \mathcal{L}_{u}\left(\mathbf{u}, \mathbf{v}_{x}, \mathbf{v}_{y}\right)=\frac{\gamma}{2}\|\mathbf{H u}-\mathbf{g}\|^{2}+\Phi\left(\mathbf{v}_{x}, \mathbf{v}_{y}\right)+ \\
& \frac{\alpha}{2}\left\|\mathbf{D}_{x} \mathbf{u}-\mathbf{v}_{x}-\mathbf{a}_{x}\right\|^{2}+\frac{\alpha}{2}\left\|\mathbf{D}_{y} \mathbf{u}-\mathbf{v}_{y}-\mathbf{a}_{y}\right\|^{2}
\end{aligned}
$$

and solving it with an iterative algorithm:

$$
\begin{aligned}
& \text { Algorithm: } \hat{\mathbf{u}}=u \text {-step }\left(\mathbf{u}^{0}\right) \\
& \text { 1: Set } \mathbf{v}_{x}^{0}=\mathbf{v}_{y}^{0}=\mathbf{a}_{x}^{0}=\mathbf{a}_{y}^{0}=0 \text { and } j=0 \\
& \text { 2: repeat } \\
& \text { 3: } \quad \mathbf{u}^{j+1}=\arg \min _{\mathbf{u}} \mathcal{L}_{u}\left(\mathbf{u}, \mathbf{v}_{x}^{j}, \mathbf{v}_{y}^{j}\right) \Longleftrightarrow \\
& {\left[\mathbf{H}^{T} \mathbf{H}+\frac{\alpha}{\gamma}\left(\mathbf{D}_{x}^{T} \mathbf{D}_{x}+\mathbf{D}_{y}^{T} \mathbf{D}_{y}\right)\right] \mathbf{u}^{j+1}=\mathbf{H}^{T} \mathbf{g}+} \\
& \frac{\alpha}{\gamma}\left[\mathbf{D}_{x}^{T}\left(\mathbf{v}_{x}^{j}+\mathbf{a}_{x}^{j}\right)+\mathbf{D}_{y}^{T}\left(\mathbf{v}_{y}^{j}+\mathbf{a}_{y}^{j}\right)\right] \\
& \text { 4: } \quad\left\{\mathbf{v}_{x}^{j+1}, \mathbf{v}_{y}^{j+1}\right\}=\arg \min _{\mathbf{v}_{x}, \mathbf{v}_{x}} \mathcal{L}_{u}\left(\mathbf{u}^{j+1}, \mathbf{v}_{x}, \mathbf{v}_{y}\right) \Longleftrightarrow \\
& {\left[\mathbf{v}_{x}^{j+1}\right]_{i}=\left[\mathbf{D}_{x} \mathbf{u}^{j+1}-\mathbf{a}_{x}^{j}\right]_{i}[\mathbf{s}]_{i}^{-1} \max \left([\mathbf{s}]_{i}-\frac{1}{\alpha}, 0\right),} \\
& {\left[\mathbf{v}_{y}^{j+1}\right]_{i}=\left[\mathbf{D}_{y} \mathbf{u}^{j+1}-\mathbf{a}_{y}^{j}\right]_{i}[\mathbf{s}]_{i}^{-1} \max \left([\mathbf{s}]_{i}-\frac{1}{\alpha}, 0\right),} \\
& \text { where } \\
& {[\mathbf{s}]_{i}=\sqrt{\left[\mathbf{D}_{x} \mathbf{u}^{j+1}-\mathbf{a}_{x}^{j}\right]_{i}^{2}+\left[\mathbf{D}_{y} \mathbf{u}^{j+1}-\mathbf{a}_{y}^{j}\right]_{i}^{2}}} \\
& \text { 5: } \quad \mathbf{a}_{x}^{j+1}=\mathbf{a}_{x}^{j}-\mathbf{D}_{x} \mathbf{u}^{j+1}+\mathbf{v}_{x}^{j+1} \\
& \mathbf{a}_{y}^{j+1}=\mathbf{a}_{y}^{j}-\mathbf{D}_{y} \mathbf{u}^{j+1}+\mathbf{v}_{y}^{j+1} \\
& \text { 6: } \quad j \leftarrow j+1 \\
& \text { 7: until stopping criterion is satisfied } \\
& \text { 8: return } \hat{\mathbf{u}} \leftarrow \mathbf{u}^{j}
\end{aligned}
$$

The iterative algorithm consists of three update steps: lines 3, 4, and 5. Variables $\mathbf{a}_{x}$ and $\mathbf{a}_{y}$ are introduced by the ALM. Their update on line 5 is trivial. It is worth drawing a relation of the ALM to a penalty method. If we omit the updating step for $\mathbf{a}_{x}$ and $\mathbf{a}_{y}$, and keep $\mathbf{a}_{x}=\mathbf{a}_{y}=0$, the above algorithm defaults to the penalty method. The penalty method converges to the solution of constrained problem (23) only if we keep increasing $\alpha$ to infinity while iterating as advocated in [30]. This is however not practical as the problem becomes gradually more ill-posed with increasing $\alpha$. This drawback is avoided in the ALM. Since $\Phi$ is a lower semicontinuous, proper, convex function ${ }^{2}$, and $\left[\mathbf{D}_{x}^{T}, \mathbf{D}_{y}^{T}\right]^{T}$ has full column rank then, if (23) has a solution, the $u$-step algorithm converges to this solution even for $\alpha$ relatively small and fixed. This important theorem was proved in [31].

Since $\mathcal{L}_{u}$ in (24) is quadratic with respect to $\mathbf{u}$, minimization on line 3 is a solution to a set of linear equations. We show later that this can be solved efficiently in the Fourier domain.

\footnotetext{
${ }^{2}$ In our case, $\Phi$ is continuous and thus lower semicontinuous
} 


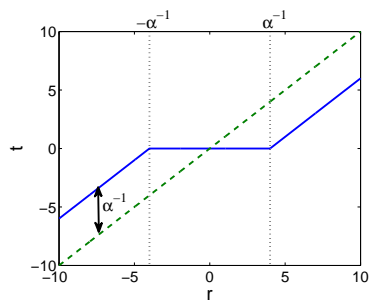

(a)

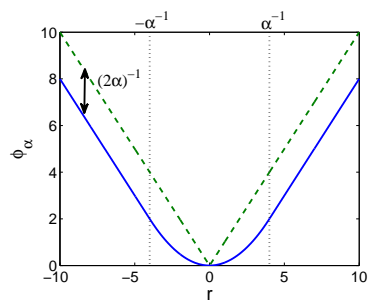

(b)
Fig. 7. Soft thresholding: (a) shrinkage formula (26) for a nonzero threshold $1 / \alpha$ (solid) and for $1 / \alpha=0$ (dashed); (b) corresponding $\phi_{\alpha}$ in (25) for a nonzero threshold $1 / \alpha$ (solid) and for $1 / \alpha=0$ (dashed). Note that $\phi_{\alpha}$ is a relaxed form of the $\ell_{1}$ norm, which is the absolute value (dashed) in this simple case.

The beauty of variable splitting is that minimization with respect to $\mathbf{v}_{x}$ and $\mathbf{v}_{y}$ is, by definition, the Moreau proximal mapping [32] of $\Phi$ applied to $\mathbf{D}_{x} \mathbf{u}^{j+1}-\mathbf{a}_{x}^{j}$ and $\mathbf{D}_{y} \mathbf{u}^{j+1}-\mathbf{a}_{y}^{j}$. The problem can be solved for each $i$-th element independently. Let $\mathbf{t}=\left[\left[\mathbf{v}_{x}\right]_{i},\left[\mathbf{v}_{y}\right]_{i}\right]^{T}$ and $\mathbf{r}=\left[\left[\mathbf{D}_{x} \mathbf{u}-\mathbf{a}_{x}\right]_{i},\left[\mathbf{D}_{y} \mathbf{u}-\mathbf{a}_{y}\right]_{i}\right]^{T}$ be vectors of size $2 \times 1$, the problem on line 4 is of the form

$$
\phi_{\alpha}(\mathbf{r})=\min _{\mathbf{t}}\left(\frac{\alpha}{2}\|\mathbf{r}-\mathbf{t}\|^{2}+\|\mathbf{t}\|\right)
$$

and as proved in [30] the minimum is reached for

$$
\mathbf{t}=\frac{\mathbf{r}}{\|\mathbf{r}\|} \max \left(\|\mathbf{r}\|-\frac{1}{\alpha}, 0\right),
$$

which is a generalized shrinkage formula for vectors. For $\mathbf{r}$ scalar, (26) corresponds to a well known soft-thresholding formula plotted as solid line in Fig. 7(a). It is interesting to note that after substituting for $\mathbf{t}$ in (25), $\phi_{\alpha}(\mathbf{r})$ (solid line in Fig. 7(b)) can be written in a closed form

$$
\phi_{\alpha}(\mathbf{r})= \begin{cases}\frac{\alpha}{2}\|\mathbf{r}\|^{2} & \text { if }\|\mathbf{r}\|<\frac{1}{\alpha} \\ \|\mathbf{r}\|-\frac{1}{2 \alpha} & \text { otherwise },\end{cases}
$$

which is a relaxed form of the original $\phi(\mathbf{r})=\|\mathbf{r}\|$ in the isotropic TV definition (6). If $\alpha \rightarrow \infty$ then $\phi_{\alpha} \rightarrow \phi$ and the corresponding graphs are plotted as dashed lines in Fig. 7.

\section{B. H-step}

The kernel estimation proceeds analogously to the $u$-step. Using the proposed regularizer (20), minimization with respect to the PSFs (5) writes as

$$
\min _{\mathbf{h}} \frac{\gamma}{2}\|\mathbf{U h}-\mathbf{g}\|^{2}+\frac{\delta}{2} \mathbf{h}^{T} \mathbf{R}_{\Delta} \mathbf{h}+\Psi(\mathbf{h})
$$

Applying variable splitting $\mathbf{w}=\mathbf{h}$ yields a constrained problem

$$
\min _{\mathbf{h}, \mathbf{w}} \frac{\gamma}{2}\|\mathbf{U h}-\mathbf{g}\|^{2}+\frac{\delta}{2} \mathbf{h}^{T} \mathbf{R}_{\Delta} \mathbf{h}+\Psi(\mathbf{w}) \quad \text { s.t. } \quad \mathbf{w}=\mathbf{h},
$$

Then we consider a functional

$\mathcal{L}_{h}(\mathbf{h}, \mathbf{w})=\frac{\gamma}{2}\|\mathbf{U h}-\mathbf{g}\|^{2}+\frac{\delta}{2} \mathbf{h}^{T} \mathbf{R}_{\Delta} \mathbf{h}+\Psi(\mathbf{w})+\frac{\beta}{2}\|\mathbf{h}-\mathbf{w}-\mathbf{b}\|^{2}$

and solve it with an iterative algorithm:

Algorithm: $\hat{\mathbf{h}}=h$-step $\left(\mathbf{h}^{0}\right)$

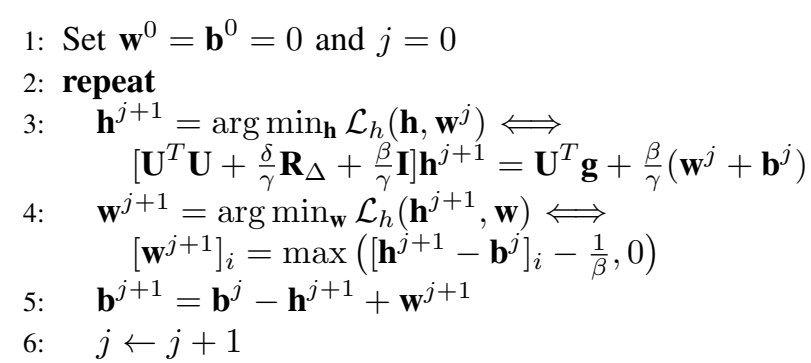

7: until stopping criterion is satisfied

8: return $\hat{\mathbf{h}} \leftarrow \mathbf{h}^{j}$

Matrix I denotes identity of size $K \hat{L} \times K \hat{L}$. As in the $u$-step, the $h$-step iterative algorithm consists of three update steps: lines 3, 4, and 5. Since $\mathcal{L}_{h}$ in (30) is quadratic with respect to $\mathbf{h}$, minimization on line 3 is a solution to a set of linear equations. Minimization with respect to $\mathbf{w}$ is again the Moreau proximal mapping, this time, of $\Psi$ applied to $\mathbf{h}^{j+1}-\mathbf{b}^{j}$ and it is solved element-wise. Let $t=[\mathbf{w}]_{i}$ and $r=[\mathbf{h}-\mathbf{b}]_{i}$, the problem on line 4 is of the form

$$
\psi_{\beta}(r)=\min _{t}\left(\frac{\beta}{2}(r-t)^{2}+\psi(t)\right),
$$

where $\psi$ is our positivity-sparsity enforcing function defined in (21) and plotted as dashed line in Fig. 8(b). After some manipulation, one can see that the minimum is reached for

$$
t=\max \left(r-\frac{1}{\beta}, 0\right),
$$

The plot of this "one-sided" thresholding function is in Fig. 8(a), solid line. Using the thresholding function, a closed form of $\psi_{\beta}$ is

$$
\psi_{\beta}(r)= \begin{cases}\frac{\beta}{2} r^{2} & \text { if } r<\frac{1}{\beta} \\ r-\frac{1}{2 \beta} & \text { otherwise }\end{cases}
$$

with a plot in Fig. 8(b), solid line. The function linearly increases in the positive domain, while in the negative domain it increases quadratically. If $\beta \rightarrow \infty$ then $\psi_{\beta} \rightarrow \psi$ and the thresholding function in (32) approaches the dashed line in Fig. 8(a). However as in the $u$-step, we do not need to increase $\beta$ to infinity for the $h$-step algorithm to converge to the solution of the constrained problem (29). The ALM approach with its extra variable b converges. Note, that $\psi$ must be a lower semicontinuous, proper, convex function for the method to converge, which is the case. Interestingly, if we replaced in the definition (21) infinity with some large but finite number, the resulting function would not be convex any more. Infinity in the definition might look dangerous but it turns out to give an elegant solution in the form of thresholding function (32).

\section{Implementation}

We have analyzed the main points ( $u$ and $h$ step) of the optimization algorithm. Now we proceed with the description of algorithm's main loop and computational cost of individual steps. Let $N$ denote the number of pixels in the output image $u$ and $\hat{L}$ the number of pixels in our overestimated PSF support. The main loop of the MC blind deconvolution alternating minimization algorithm looks as follows: 


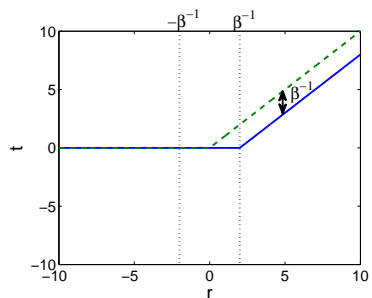

(a)

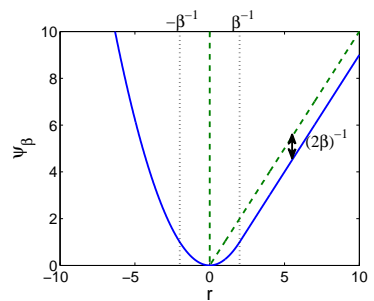

(b)
Fig. 8. Thresholding in the blur domain: (a) shrinkage formula (32) for a nonzero threshold $1 / \beta$ (solid) and for $1 / \beta=0$ (dashed); (b) corresponding $\psi_{\beta}$ in (31) for a nonzero threshold $1 / \beta$ (solid) and for $1 / \beta=0$ (dashed).

\section{MC blind deconvolution}

Require: $K \geq 2$ input images $\left\{\mathbf{g}_{k}\right\}$; blur size $\hat{L}$; parameters $\alpha, \beta, \delta, \gamma$

1: Set $j=0, \hat{\mathbf{h}}_{k}^{0}$ 's to delta functions, and $\hat{\mathbf{u}}^{0}=0$

2: Calculate $\mathbf{R}_{\Delta}$

3: repeat

4: $\quad \hat{\mathbf{u}}^{j+1}=u$-step $\left(\hat{\mathbf{u}}^{j}, \hat{\mathbf{h}}^{j}\right)$

5: $\quad \hat{\mathbf{h}}^{j+1}=h$-step $\left(\hat{\mathbf{u}}^{j+1}, \hat{\mathbf{h}}^{j}\right)$

6: $\quad j \leftarrow j+1$

7: until stopping criterion is satisfied

8: return $\hat{\mathbf{u}} \leftarrow \hat{\mathbf{u}}^{j}$

The stopping criterion, which we typically use, is $\| \hat{\mathbf{h}}^{j}-$ $\hat{\mathbf{h}}^{j-1}\|/\| \hat{\mathbf{h}}^{j} \|<$ tol. The same can be used in the $h$-step and likewise in the $u$-step using $\mathbf{u}$ instead of $\mathbf{h}$. Calculation of $\mathbf{R}_{\Delta}$ can be done using the Fast Fourier Transform (FFT) without explicitly constructing the convolution matrices $\mathbf{G}_{k}$ 's. Since $\mathbf{G}_{k}$ 's are "valid" convolutions, we can construct only one row of $\mathbf{R}_{\Delta}$ at a time and the overall complexity is thus $O(K \hat{L} N \log N)$.

In general, the most time consuming is the $u$-step, which requires an inversion of the huge $N \times N$ matrix $\left[\mathbf{H}^{T} \mathbf{H}+\right.$ $\left.\frac{\alpha}{\gamma}\left(\mathbf{D}_{x}^{T} \mathbf{D}_{x}+\mathbf{D}_{y}^{T} \mathbf{D}_{y}\right)\right]$. One can apply iterative solvers, such as conjugate gradient, to avoid direct inversion, but we can do even better and have a direct solver. In our formulation, $\mathbf{H}, \mathbf{D}_{x}$, and $\mathbf{D}_{y}$ are convolution matrices. To avoid any ringing artifacts close to image boundaries, they should perform "valid" convolution, i.e., the output image is smaller and covers a region where both the input image and convolution kernel are fully defined. If we properly adjust the image borders, by using for example function edgetaper in MATLAB, we can replace "valid" convolution with block-circulant one and ringing artifacts will be almost undetectable. The TV regularizer also helps to reduce such artifacts. FFT diagonalizes block-circulant convolution matrices and inversion is thus straightforward. The remaining update steps for $\mathbf{v}_{x}\left(\mathbf{v}_{y}\right)$ and $\mathbf{a}_{x}\left(\mathbf{a}_{y}\right)$ are simple and can be computed in $O(N)$ time. The $u$-step is thus carried out with overall $O(N \log N)$ cost.

Unlike the $u$-step, which is calculated almost entirely in the Fourier domain, we perform the $h$-step in the image domain, since we need the constrained kernel support $\hat{L}$. Otherwise, $\mathbf{R}_{\Delta}$ becomes a very uninformative regularizer as explained in Sec. IV-C. On line 3 of the $h$-step algorithm, we have to invert the matrix $\left[\mathbf{U}^{T} \mathbf{U}+\frac{\delta}{\gamma} \mathbf{R}_{\Delta}+\frac{\beta}{\gamma} \mathbf{I}\right]$, which is of size $K \hat{L} \times K \hat{L}$ and thus much smaller than the matrix in the $u$-step. Typically, the size of blurs is not more than $40 \times 40$ pixels $(L=1600)$ and for two input images $(K=2)$ the matrix size is $3200 \times$ 3200 , which is still relatively small ${ }^{3}$. One can again apply an iterative solver such as conjugate gradient, but we found it much more efficient to store the whole matrix and perform Cholesky decomposition to solve this problem. This can be computed in $O\left((K \hat{L})^{3}\right)$ time. Update steps for $\mathbf{w}$ and $\mathbf{b}$ are again very simple and require $O(K \hat{L})$ operations.

Setting parameters is based solely on our empirical studies and cannot be considered as a rigorous procedure. The optimization method has four parameters. We have noticed that in general they can be fixed relative to one of them, $\gamma$, which depends on the noise level. This observation is not superficial. Authors in [5] (as well as is [4] for the split Bregman method) also recommend to set parameters introduced by the ALM, in our case $\alpha$ and $\beta$, with respect to the weight $\gamma$ of the fidelity term. Parameter $\delta$, which is the weight of the MC constraint term $\mathbf{h}^{T} \mathbf{R}_{\Delta} \mathbf{h}$, is proportional to the noise variance as shown in (16) and therefore should be fixed to $\gamma$ as well. The role of thumb is to set $\gamma$ equal to a ratio of signal and noise variances, i.e., $\mathrm{SNR}=50 \mathrm{~dB} \Rightarrow \gamma=10^{5}$ or SNR $=20 \mathrm{~dB} \Rightarrow$ $\gamma=10^{2}$, etc. ${ }^{4}$ Then we have found that choosing $\alpha=10^{-1} \gamma$, $\beta=10^{4} \gamma, \delta=10^{3} \gamma$ usually results in good convergence. For higher noise levels (smaller $\gamma$ ) we observed that $\delta=10^{2} \gamma$ is better.

In our experiments, the number of iteration in the main loop, and in the $u$-step and $h$-step, typically did not exceed 10. In order to further decrease computational time, we tried to modify the algorithm in several ways. For example, we found it very effective to divide the algorithm into two stages. In the first stage, we select a small (typically $256 \times 256$ ) central region from input images and run the algorithm on this selection. In the second stage, we take the estimated PSFs from the first stage and apply one $u$-step on the whole image in order to obtain the final reconstructed image. The usable output of the first stage are thus PSFs and not the reconstructed central region. We observed that fixing $\gamma$ to 10 (even for SNR above $10 \mathrm{~dB}$ ) in the first stage and setting other parameters according to formulas as shown above produces accurate PSFs in a more reliable way. This convergence boost can be explained by noting that the reconstructed image for lower $\gamma$ becomes more piece-wise constant (patchy) with only strong edges preserved, which makes the $h$-step in the fidelity term focus only on areas around strong edges and neglect areas with details that are prone to noise.

Another modification, which proved to be a minor improvement, was to estimate PSFs in a multi-scale fashion. Initializing with upsampled PSFs from the courser levels tend to decrease number of iterations. However, we observed that more than 2 levels (half-sized and original scale) are not necessary and that the choice of upsampling algorithm is important. Simple linear upsampling generates PSFs that are wider than the true PSFs on that scale and we waste several iterations of the algorithm to shrink the PSFs back. In our

\footnotetext{
${ }^{3} \mathrm{~A}$ matrix of such size, if stored in double precision, occupies approximately $78 \mathrm{MB}$ of memory, which current computers can easily handle.

${ }^{4} \mathrm{We}$ use a standard definition of the signal to noise ratio, SNR = $10 \log \left(\frac{s^{2}}{\sigma^{2}}\right)$, where $s^{2}$ and $\sigma^{2}$ are the signal and noise variances, respectively.
} 
tests, we were using a Lanczos interpolation method, which seems to give the best results.

To provide the cost of individual steps in terms of computer time, we performed blind deconvolution of two 1 Mpixel images with PSF size $40 \times 40$ on a $2.7 \mathrm{GHz}$ Pentium DualCore CPU using our MATLAB implementation. The cost of one iteration inside the $u$-step and $h$-step is around $0.8 \mathrm{~s}$ and $4.5 \mathrm{~s}$, respectively. Calculating the matrix $\mathbf{R}$ using the whole images, takes in this case 11 minutes, which is clearly the most time consuming step. However as pointed out earlier, we can calculate $\mathbf{R}$ on a small region. For example for $256 \times 256$ block, the calculation (same PSF size $40 \times 40$ ) then takes around 30 s.

\section{EXPERIMENTS}

In order to illustrate the favorable convergence properties of the proposed algorithm, we performed two sets of experiments. The first set works with synthetically blurred data and compares convergence and quality of PSF and image estimation for different SNRs and blur sizes. The second set of experiments compares the proposed algorithm with another multichannel blind deconvolution method of Katkovnik et al. [33] and demonstrates deconvolution of real photos taken with a standard digital camera.

The setup for the synthetic data experiment was the following. We took the Lena image, Fig. 9(a), and convolve it with two $9 \times 9$ blurs, Fig. 9(b), and add noise at three different levels, $\mathrm{SNR}=50,30,10 \mathrm{~dB}$. An example of blurry images for the least noisy case is in Fig. 9(c). To evaluate performance in every iteration $j$ of the main loop, we use normalized root mean square error defined as NRMSE $=\left\|\hat{\mathbf{h}}^{j}-\mathbf{h}^{*}\right\| /\left\|\mathbf{h}^{*}\right\|$, where $\hat{\mathbf{h}}^{j}$ is the estimation of PSFs after $j$ iterations and $\mathbf{h}^{*}$ are the true PSFs. NRMSE as a function of iterations and estimated PSFs for different situations are summarized in Fig. 10. NRMSE is plotted in logarithmic scale. Three graphs correspond to three levels of SNRs. In each case we ran the algorithm with three different PSF supports: $9 \times 9$ (solid line), $15 \times 15$ (dotted line) and $21 \times 21$ (dashed line). The corresponding estimated sharp images for PSF support $21 \times 21$ are summarized in Fig. 11. One can see that the proposed method provides accurate results regardless of the degree of PSF size overestimation and shows robustness with respect to noise.

There are several interesting points we can draw from the obtained results. First of all, the mean square error (MSE) decreases very quickly. In most of the cases, after 5 iterations MSE remains almost constant. For overestimated blur supports (dotted and dashed line) MSE reaches almost the same level as for the correct blur support (solid line), but the decrease is slightly less sharp (particularly visible for $\mathrm{SNR}=50 \mathrm{~dB}$ ). This is logical, since in the overestimated case the dimensionality of the problem is higher and the MC constraint $\mathbf{R}_{\Delta}$ is less effective as discussed in Sec. IV-C. Clearly, as the noise level increases, the lowest attainable MSE increases as well. For SNR $=50 \mathrm{~dB}$, Fig. 10(a), estimated PSFs are very accurate. The corresponding estimated image in Fig. 11(a) is almost perfect. For SNR $=30 \mathrm{~dB}$, Fig. 10(b), the estimated PSFs take the shape of the true PSFs but are slightly blurred. The estimated image in Fig. 11(b) still looks very sharp and artifact-free. As the noise level increases further to SNR $=10 \mathrm{~dB}$, Fig. 10(c), the quality of deconvolution starts to deteriorate but the TV denoising feature of the method is evident as seen in Fig. 11(c).

There is very little in the literature to which we can directly compare, which uses multiple frames in the process. Most of the multichannel work presented in the introduction is mainly theoretical and present no algorithms for large scale problems. Comparing to single-channel results is possible, but we do not feel that this is fair to these other methods. To our knowledge the only recent method, which is intrinsically multichannel and claims to work with large kernels, was proposed by Katkovnik et al. [33]. This method performs alternating minimization by switching between minimization with respect to the image (corresponds to our $u$-step) and minimization with respect to the kernels (corresponds to our $h$-step). A variation of the steepest descent algorithm is used for minimization. Everything is implemented in the Fourier domain as in our case. For minimization, we use ALM in order to work with nonlinear regularization terms in an efficient manner. Katkovnik et al. use a variation of the steepest descent algorithm with only quadratic terms. Instead of using regularization, they project current estimation after every iteration into an admissible set of solutions (such as positive PSFs with limited support and image intensity values between 0 and 1 ) and perform spatially adaptive image denoising based on intersection of confidence intervals (ICI) rule. To compare the methods, we took a data set generated by Levin et al. [15], which contains 4 images blurred by 8 PSFs providing 32 blurred images; see Fig. 12(ab). The blurred images are real and captured by a digital camera. The ground truth PSFs in Fig. 12(b) were estimated by a collection of point sources installed in the observed scene. We divided the blurred images into 8 groups (each containing one image blurred by 4 blurs) and applied both methods. NRMSE of the estimated images and blurs are plotted in Fig. 12(c-d). One can see that in half of the cases our method provides better PSFs (in the NRMSE sense) and outperforms the other method in the image NRMSE in all 8 cases. In addition, our method requires only 10 iterations of alternating minimization whereas the other method requires roughly 100 iterations to achieve these results. ${ }^{5}$

In order to demonstrate, that the algorithm works well in many practical applications, we took several pairs of images with a 3Mpixel digital camera Olympus C3020Z and applied the proposed algorithm. Light conditions were low and the shutter speed of the camera was typically longer than $1 / 10$ s. Such setting produces nice blurry images, when the camera is held in hands. It is of course necessary to first register the input photos before the algorithm can be applied. In our case, we do not have to deal with heavily misregistered data, since the images were take one after another with a minimum delay. A fast registration method, which proved to be adequate and was used in these experiments, works as follows. A reference image is selected from the input set $\left\{\mathbf{g}_{k}\right\}$ and the other

\footnotetext{
${ }^{5}$ It is true that we perform at most 10 iterations inside both $u$-step and $h$ step. Katkovnik's method cannot perform many iterations inside their $u$-step and $h$-step, since they need to project into the admissible set frequently and so they do 10 steps of steepest descent in the $h$-step and 1 step in the $u$-step.
} 


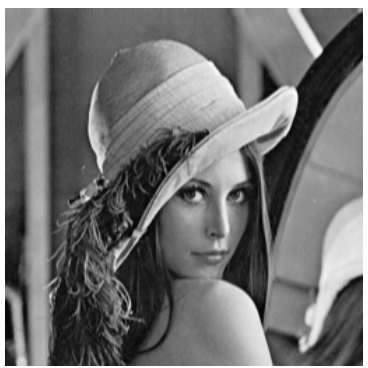

(a)

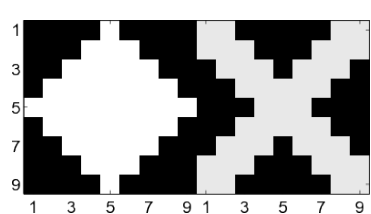

(b)

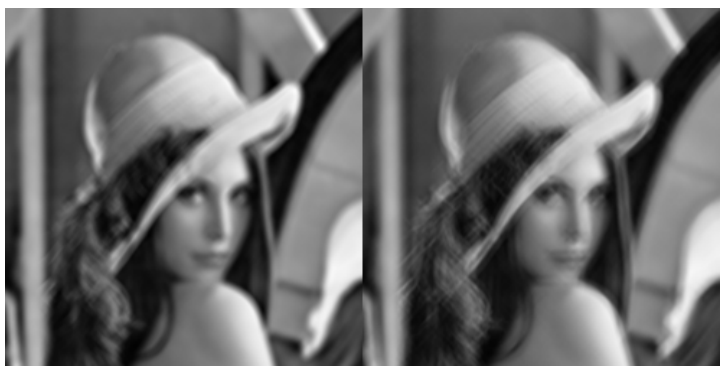

(c)

Fig. 9. Test data set: (a) original image $256 \times 256$, (b) two blurs $9 \times 9$, (c) example of an input blurry pair with SNR $=50 \mathrm{~dB}$.

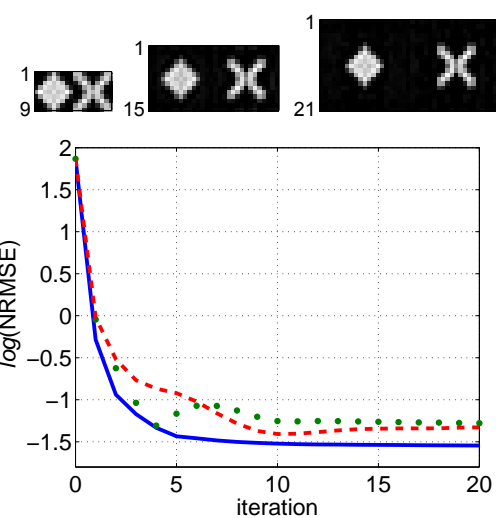

(a) $50 \mathrm{~dB}$

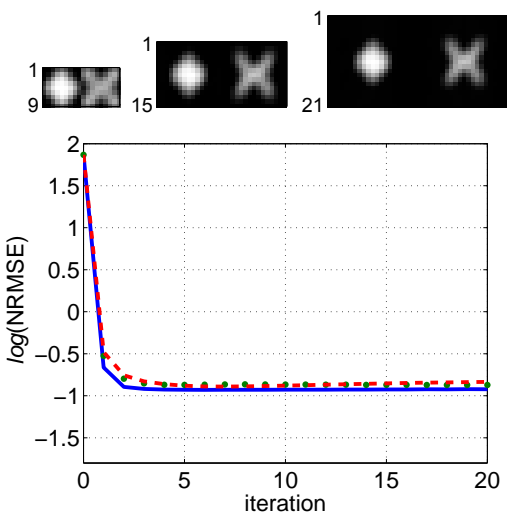

(b) $30 \mathrm{~dB}$
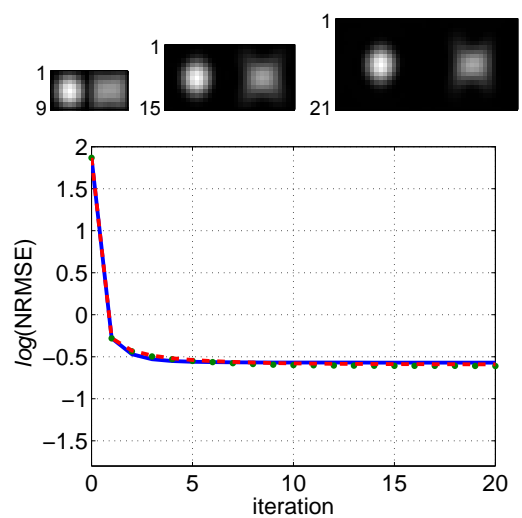

(c) $10 \mathrm{~dB}$

Fig. 10. Estimated PSFs and plots of normalized root mean square errors for different noise levels in input blurry images: (a) 50dB, (b) 30dB, (c) 10dB Three different PSF supports were considered in each noisy case: correct PSF size $9 \times 9$ (solid line), and two overestimated sizes $15 \times 15$ (dotted line) and $21 \times 21$ (dashed line).
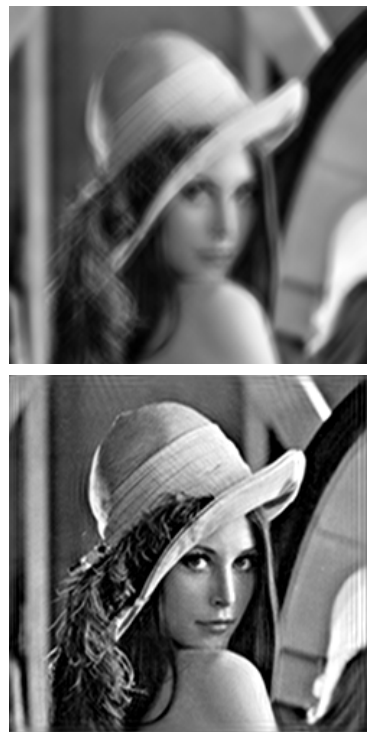

(a) $50 \mathrm{~dB}$
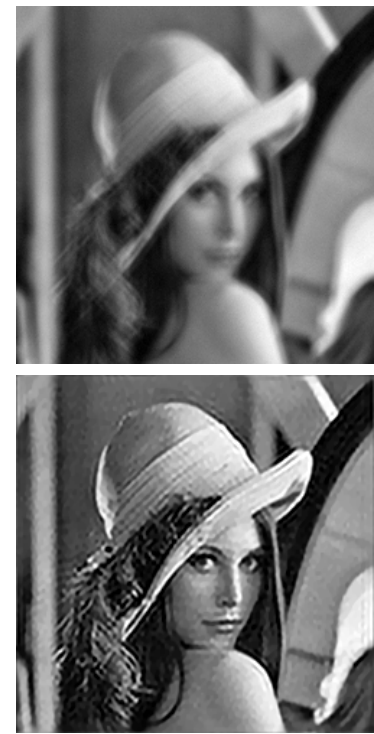

(b) $30 \mathrm{~dB}$
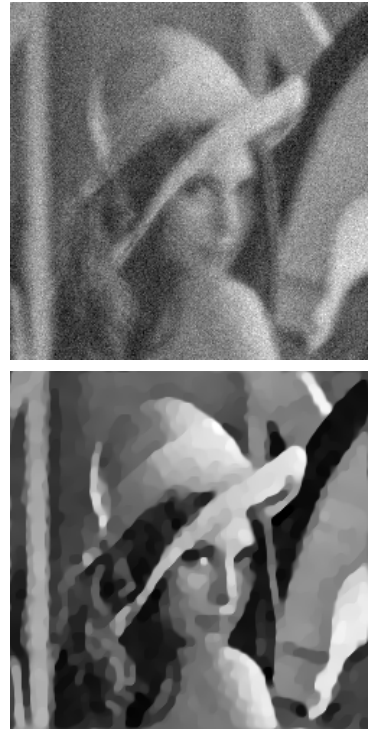

(c) $10 \mathrm{~dB}$

Fig. 11. Estimated sharp images for the PSF size set to $21 \times 21$ and three different noise levels: (a) $50 \mathrm{~dB}$, (b) $30 \mathrm{~dB}$, (c) $10 \mathrm{~dB}$. Results are arranged as in Fig. 10. The first row shows one of the input images and the second row shows the estimated image. 


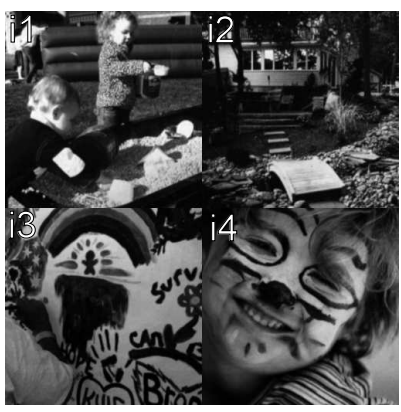

(a)

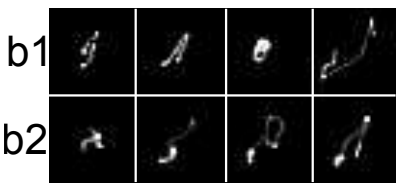

(b)

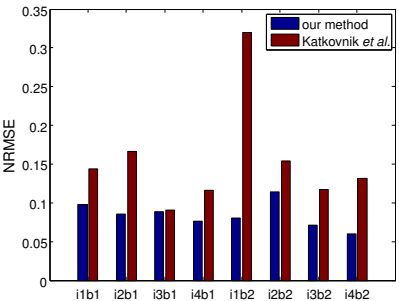

(c)

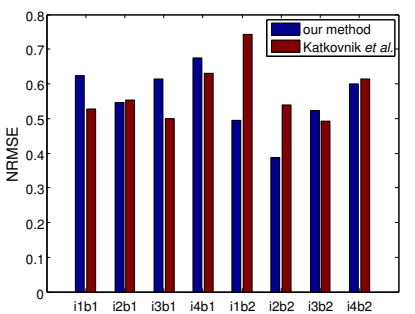

(d)

Fig. 12. Comparison with Katkovnik et al. [33]: Ground truth data from Levin's data set [15] (a) 4 images and (b) 8 blur kernels, which generates 32 blurred images. We split the kernels into two groups (b1, b2) and got 8 input sets each containing 4 blurred images. (c) shows NRMSE of estimated sharp images and (d) NRMSE of estimated kernels. Left bars are results of our method and right bars are results of [33].

images (called sensed images) are sequentially registered to the reference one. The reference and sensed image is first divided into several non-overlapping blocks (typically $6 \times 6$ ). Phase correlation is applied in each block to determine integer translation vector between the reference and the sensed block. The estimated shifts $(6 * 6=36)$ are used to calculate parameters of an affine transform. The sensed images are then interpolated using the estimated affine transforms.

Reconstruction results for two different data sets are in Figs. 13 and 14. Input image pairs exhibit relatively large blurring but the reconstructed images are sharp and with negligible artifacts (see image close-ups for better visual comparison). Estimated PSF pairs very well model motion blurs induced by camera shake. Some artifacts are visible in the second data set (Fig. 14(c)) around the snow heap in the left bottom corner. It is very likely, that the blur is slightly different in this part due to a different distance from the camera or due to rotational movement during acquisition. Since our method assumes space-invariant blurs, such artifact are however inevitable.

\section{CONCLUSION}

We have presented a new algorithm for solving multichannel blind deconvolution. The proposed approach starts by defining an optimization problem with image and blur regularization terms. To force sparse image gradients, the image regularizer is formulated using a standard isotropic total variation. The PSF regularizer consists of two terms: MC constraint (matrix $\mathbf{R}_{\Delta}$ ) and sparsity-positivity. The MC constraint is improved by considering image Laplacian, which brings better noise robustness at little cost. Positivity helps the method to convergence to a correct solution, when the used PSF size is much larger than the true one. The proposed approach solves the optimization problem in an iterative way by alternating between minimization with respect to the image ( $u$-step) and with respect to the PSFs ( $h$-step). Sparsity and positivity imply nonlinearity, but by using the variable splitting and augmented Lagrangian method (or split-Bregman method) we can solve each step efficiently and moreover convergence of each step is guaranteed. Experiments on synthetic data illustrate fast convergence of the algorithm, robustness to noise, and stability in the case of overestimated PSF sizes. Experiments on large real data underline practical aspects of the algorithm. Current and future work involves extending this approach to the spacevariant blur and analyzing the convergence properties.

\section{REFERENCES}

[1] A. Levin, R. Fergus, F. Durand, and W. T. Freeman, "Image and depth from a conventional camera with a coded aperture," ACM Trans. Graph., vol. 26, no. 3, p. 70, 2007.

[2] M. Elad, P. Milanfar, and R. Rubinstein, "Analysis versus synthesis in signal priors," Inverse Problems, vol. 23, no. 3, pp. 947-968, 2007. [Online]. Available: http://stacks.iop.org/0266-5611/23/i=3/a=007

[3] I. W. Selesnick and M. Figueiredo, "Signal restoration with overcomplete wavelet transforms: comparison of analysis and synthesis priors," in Proceedings of SPIE, vol. 7446, 2009.

[4] T. Goldstein and S. Osher, "The split bregman method for 11-regularized problems," SIAM J. Img. Sci., vol. 2, pp. 323-343, April 2009. [Online]. Available: http://portal.acm.org/citation.cfm?id=1658384.1658386

[5] M. V. Afonso, J. M. Bioucas-Dias, and M. A. T. Figueiredo, "Fast image recovery using variable splitting and constrained optimization," IEEE Transactions on Image Processing, vol. 19, no. 9, pp. 2345-2356, 2010.

[6] G. Ayers and J.C.Dainty, "Iterative blind deconvolution method and its application," Optical Letters, vol. 13, no. 7, pp. 547-549, Jul. 1988.

[7] T. Chan and C. Wong, "Total variation blind deconvolution," IEEE Trans. Image Processing, vol. 7, no. 3, pp. 370-375, Mar. 1998.

[8] R. Molina, J. Mateos, and A. K. Katsaggelos, "Blind deconvolution using a variational approach to parameter, image, and blur estimation," IEEE Transactions on Image Processing, vol. 15, no. 12, pp. 3715-3727, Dec. 2006.

[9] P. Campisi and K. Egiazarian, Eds., Blind Image Deconvolution, Theory and Application. CRC Press, 2007.

[10] R. Fergus, B. Singh, A. Hertzmann, S. T. Roweis, and W. T. Freeman, "Removing camera shake from a single photograph," in SIGGRAPH '06: ACM SIGGRAPH 2006 Papers. New York, NY, USA: ACM, 2006, pp. 787-794.

[11] J. Miskin and D. J. MacKay, "Ensemble learning for blind image separation and deconvolution." in Advances in Independent Component Analysis, M. Girolani, Ed. Springer-Verlag, 2000.

[12] J. Jia, "Single image motion deblurring using transparency," in Proc. IEEE Conference on Computer Vision and Pattern Recognition CVPR '07, 17-22 June 2007, pp. 1-8.

[13] N. Joshi, R. Szeliski, and D. J. Kriegman, "PSF estimation using sharp edge prediction," in Proc. IEEE Conference on Computer Vision and Pattern Recognition CVPR 2008, 23-28 June 2008, pp. 1-8.

[14] Q. Shan, J. Jia, and A. Agarwala, "High-quality motion deblurring from a single image," in SIGGRAPH '08: ACM SIGGRAPH 2008 papers. New York, NY, USA: ACM, 2008, pp. 1-10.

[15] A. Levin, Y. Weiss, F. Durand, and W. Freeman, "Understanding and evaluating blind deconvolution algorithms," in Proc. IEEE Conference on Computer Vision and Pattern Recognition CVPR '09, 2009.

[16] S. Cho and S. Lee, "Fast motion deblurring," ACM Transactions on Graphics (SIGGRAPH ASIA 2009), vol. 28, no. 5, p. article no. 145, 2009. 


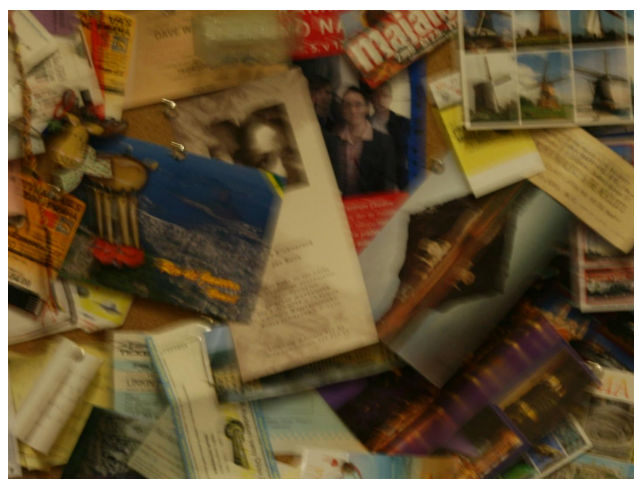

(a)

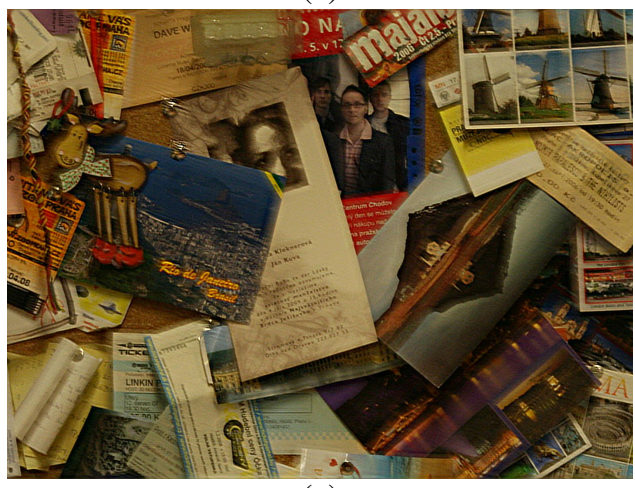

(c)

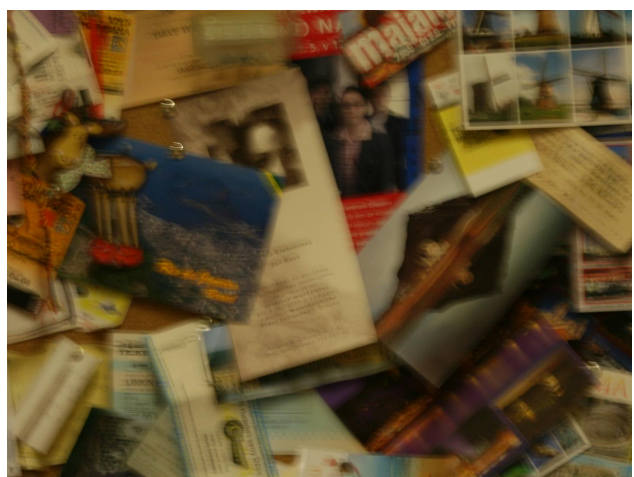

(b)
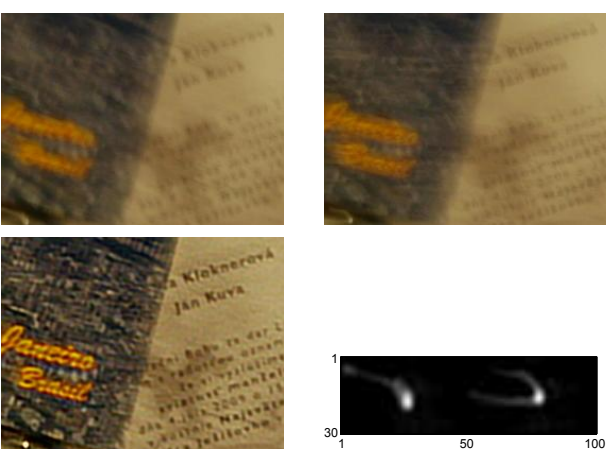

(d)

Fig. 13. Real data set: (a) - (b) two input blurry images of size $2048 \times 1536$, (c) estimated output sharp image using the proposed algorithm, (d) close-ups of the input images and the output, and estimated PSFs of size $50 \times 30$.

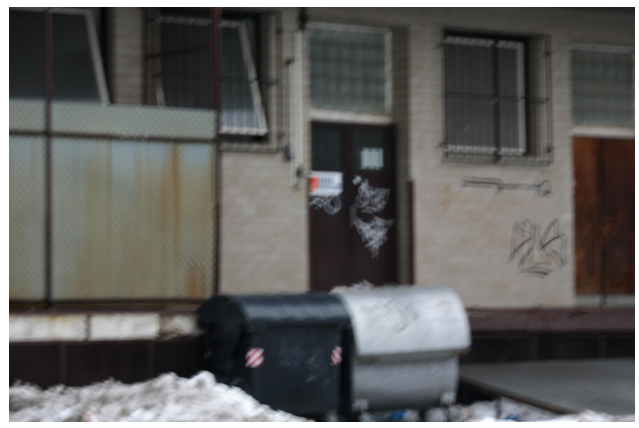

(a)

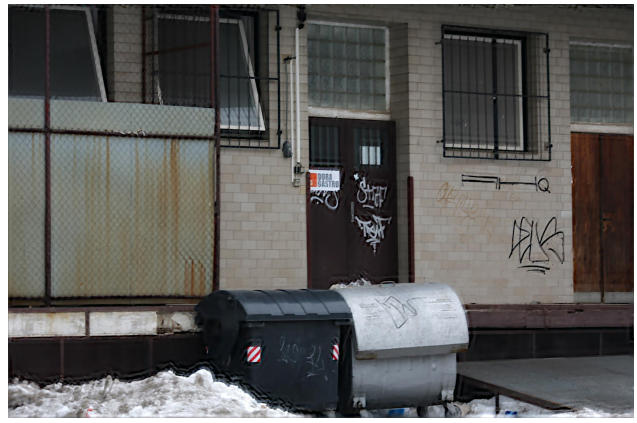

(c)

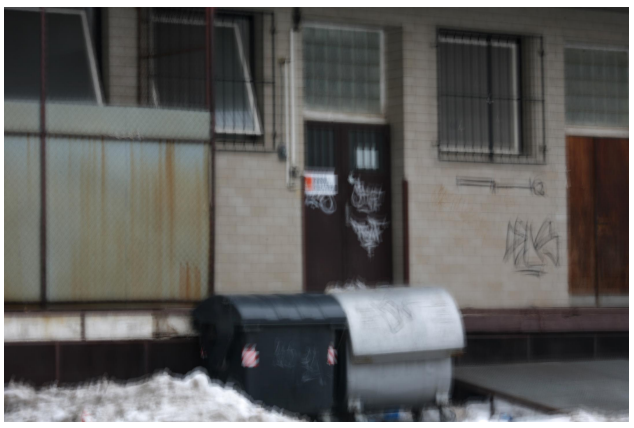

(b)
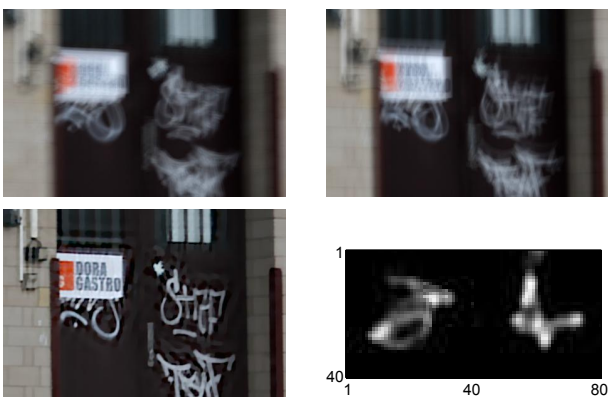

(d)

Fig. 14. Real data set: (a) - (b) two input blurry images of size $2048 \times 1536$, (c) estimated output sharp image using the proposed algorithm, (d) close-ups of the input images and the output, and estimated PSFs of size $40 \times 40$. 
[17] L. Xu and J. Jia, "Two-phase kernel estimation for robust motion deblurring," in Proceedings of the 11th European conference on Computer vision: Part I, ser. ECCV'10. Berlin, Heidelberg: Springer-Verlag, 2010, pp. 157-170. [Online]. Available: http://portal.acm.org/citation.cfm?id=1886063.1886077

[18] B. Zitová and J. Flusser, "Image registration methods: A survey," Image and Vision Computing, vol. 21, pp. 977-1000, 2003.

[19] M. Sorel and J. Flusser, "Space-variant restoration of images degraded by camera motion blur," IEEE Transactions on Image Processing, vol. 17 , no. 2, pp. 105-116, Feb. 2008.

[20] T. Schulz, "Multiframe blind deconvolution of astronomical images," $J$. Opt. Soc. Am. A, vol. 10, no. 5, pp. 1064-1073, May 1993.

[21] G. Harikumar and Y. Bresler, "Perfect blind restoration of images blurred by multiple filters: Theory and efficient algorithms," IEEE Trans. Image Processing, vol. 8, no. 2, pp. 202-219, Feb. 1999.

[22] G. Giannakis and R. Heath, "Blind identification of multichannel FIR blurs and perfect image restoration," IEEE Trans. Image Processing, vol. 9, no. 11, pp. 1877-1896, Nov. 2000.

[23] H.-T. Pai and A. Bovik, "On eigenstructure-based direct multichannel blind image restoration," IEEE Trans. Image Processing, vol. 10, no. 10, pp. 1434-1446, Oct. 2001.

[24] S. Pillai and B. Liang, "Blind image deconvolution using a robust GCD approach," IEEE Trans. Image Processing, vol. 8, no. 2, pp. 295-301, Feb. 1999.

[25] M. Haindl and S. Šimberová, "Model-based restoration of shortexposure solar images," in Frontiers in Artificial Intelligence and Applications, ser. Knowledge-Based Intelligent Engeneering Systems, L. Jain and R. Howlett, Eds., vol. 87. Amsterdam: Publisher IOS Press, 2002, pp. 697-706.

[26] G. Panci, P. Campisi, S. Colonnese, and G. Scarano, "Multichannel blind image deconvolution using the bussgang algorithm: Spatial and multiresolution approaches," IEEE Trans. Image Processing, vol. 12 , no. 11, pp. 1324-1337, Nov. 2003.

[27] F. Šroubek and J. Flusser, "Multichannel blind deconvolution of spatially misaligned images," IEEE Trans. Image Processing, vol. 14, no. 7, pp. 874-883, Jul. 2005.

[28] L. Rudin, S. Osher, and E. Fatemi, "Nonlinear total variation based noise removal algorithms," Physica D, vol. 60, pp. 259-268, 1992.

[29] J. B. Reade, Calculus with Complex Numbers. CRC Press, 2003, ch. Fundamental theorem of algebra, pp. 75-81.

[30] J. Yang, W. Yin, Y. Zhang, and Y. Wang, "A fast algorithm for edge-preserving variational multichannel image restoration," SIAM J. Img. Sci., vol. 2, pp. 569-592, May 2009. [Online]. Available: http://portal.acm.org/citation.cfm?id=1658384.1658394

[31] J. Eckstein and D. P. Bertsekas, "On the douglas-rachford splitting method and the proximal point algorithm for maximal monotone operators," Mathematical Programming, vol. 55, pp. 293-318, 1992.

[32] J. Moreau, "Proximité et dualité dans un espace hilbertien," Bulletin de la Société Mathématique de France, vol. 93, pp. 273-299, 1965.

[33] V. Katkovnik, D. Paliy, K. Egiazarian, and J. Astola, "Frequency domain blind deconvolution in multiframe imaging using anisotropic spatially-adaptive denoising," in Proc. 14th European Signal Processing Conference, EUSIPCO, September 2006.

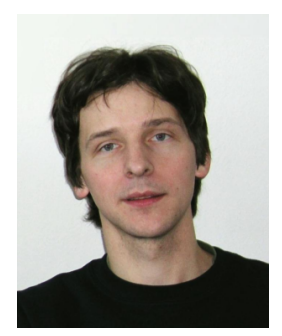

Filip Šroubek received the MS degree in computer science from the Czech Technical University, Prague, Czech Republic in 1998 and the PhD degree in computer science from Charles University, Prague, Czech Republic in 2003. From 2004 to 2006, he was on a postdoctoral position in the Instituto de Optica, CSIC, Madrid, Spain. In 2010 and 2011, he was the Fulbright Visiting Scholar at the University of California, Santa Cruz. He is currently with the Institute of Information Theory and Automation and partially also with the Institute of Photonics and Electronics, where both institutes are part of the Academy of Sciences of the Czech Republic. Filip Šroubek is an author of seven book chapters and over 80 journal and conference papers on image fusion, blind deconvolution, super-resolution, and related topics.

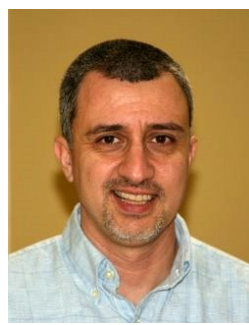

Peyman Milanfar received the BS degree in electrical engineering and mathematics from the University of California, Berkeley, in 1988, and the MS, EE, and $\mathrm{PhD}$ degrees in electrical engineering from the Massachusetts Institute of Technology, Cambridge, in 1990, 1992, and 1993, respectively. Until 1999, he was a senior research engineer at SRI International, Menlo Park, California. He is currently a professor of electrical engineering at the University of California, Santa Cruz. From 1998 to 2000, he was a consulting assistant professor of computer science at Stanford University, California, where he was also a visiting associate professor in 2002. His research interests include statistical signal, image processing, and inverse problems. He won the US National Science Foundation CAREER award, and the best paper award from the IEEE Signal Processing Society in 2010. From 1998 to 2001, he was an associate editor for the IEEE Signal Processing Letters, and was an associate editor for the IEEE Transactions on Image Processing from 2005-2010. He is currently on the editorial board of the SIAM Journal of Imaging Science, and Image and Vision Computing. He is a member of the Signal Processing Society Image, Video, and Multidimensional Signal Processing (IVMSP) Technical Committee. He is a fellow of the IEEE. 